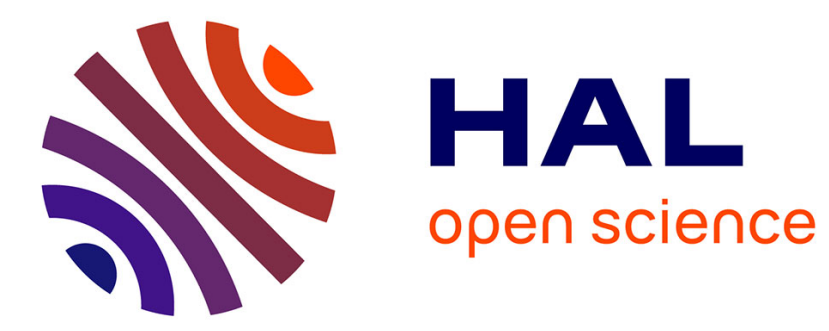

\title{
Non-linear control structures for rotorcraft positioning
}

\author{
Antoine Drouin, Thierry Miquel, Felix Mora-Camino
}

\section{To cite this version:}

Antoine Drouin, Thierry Miquel, Felix Mora-Camino. Non-linear control structures for rotorcraft positioning. AIAA GNC 2008, AIAA Guidance, Navigation and Control Conference, Aug 2008, Honolulu, United States. pp xxxx, 10.2514/6.2008-7006 . hal-00938740

\section{HAL Id: hal-00938740 \\ https://hal-enac.archives-ouvertes.fr/hal-00938740}

Submitted on 29 Apr 2014

HAL is a multi-disciplinary open access archive for the deposit and dissemination of scientific research documents, whether they are published or not. The documents may come from teaching and research institutions in France or abroad, or from public or private research centers.
L'archive ouverte pluridisciplinaire HAL, est destinée au dépôt et à la diffusion de documents scientifiques de niveau recherche, publiés ou non, émanant des établissements d'enseignement et de recherche français ou étrangers, des laboratoires publics ou privés. 


\title{
Non-Linear Control Structures for Rotorcraft Positioning
}

\author{
Antoine Drouin ${ }^{*}$ \\ ENAC, Toulouse, 31055, France \\ Thierry Miquel ${ }^{+}$ \\ DSNA/DTI/R\&D, Toulouse, 31055, France \\ Felix Mora-Camino ${ }^{\circ}$ \\ ENAC, Toulouse, 31055, France
}

The purpose of this communication is to apply and compare three different non-linear control approaches to the design of control structures with control laws allowing autonomous positioning and orientation for a four-rotor aircraft. Realistic rotorcraft flight dynamics are established and analyzed so that a reference three-layers control structure is defined. Then are introduced different non-linear control approaches: non-linear inverse control, backstepping control and differential flat control. The compatibility of these control approaches with the three-layer control structure is assessed and adaptations are .proposed so that they can cope with the guidance of the rotorcraft. The corresponding control laws are detailed and their expected performances are discussed. The simplification of these control laws around equilibrium conditions produces close quasi-linear proportional derivative controllers. A careful tuning of one of them provides a reference to evaluate the improvements resulting from the use of full non-linear control laws, when applied to the positioning and orientation problem. This evaluation is performed by simulation and numerical results are displayed for analysis.

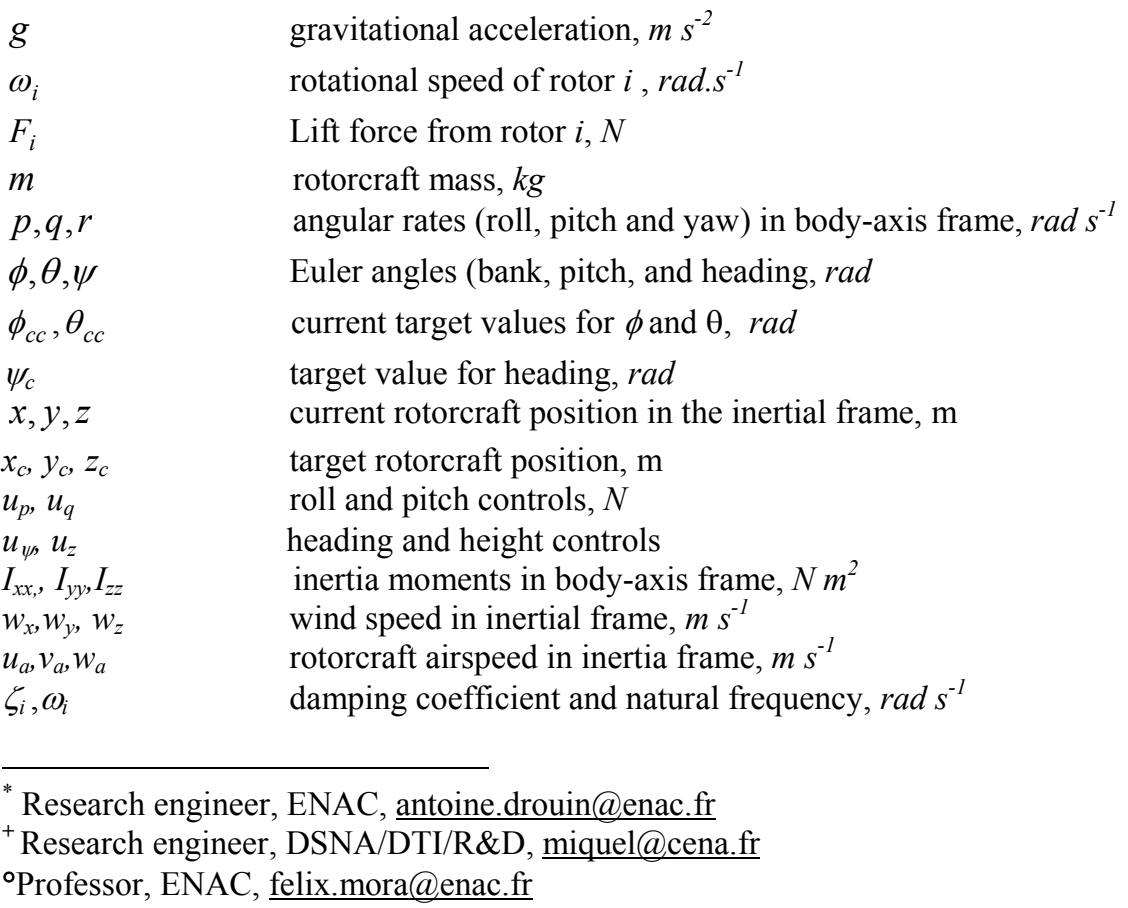




\section{Introduction}

$\mathrm{I}$

$\mathrm{n}$ the last years interest has risen for the four-rotor concept since it appears to present simultaneously hovering, orientation and trajectory tracking capabilities of interest for many practical applications [1]. The flight mechanics of this rotorcraft are highly non-linear and different control approaches (integral LQR techniques, integral sliding mode control [2], reinforcement learning [3]) have been considered with little success to achieve either only autonomous hovering and orientation or also trajectory tracking.

In this paper we consider the flight dynamics of a four- rotor aircraft with fixed pitch blades. The control problem of interest is the design of flight control laws enabling autonomous positioning and orientation for this class of rotorcraft. This study investigates the solution of this problem using three of the main popular approaches for nonlinear control law design ( non-linear inverse control, backstepping control and differential flat control). Here it is required that a single continuous control law performs the whole maneuver while to achieve it by manual control it appears necessary to go through a succession of elementary maneuvers.

An analysis of the rotorcraft flight dynamics is realized. This leads to the proposal of a reference control structure composed of three layers associated to the different time scales which made up the dynamics of he rotorcraft and to its alighted causality relationships.

The first considered non-linear control approach, non-linear inverse control, has been already applied by different authors $[4,5,6]$ to the case of aircraft trajectory tracking. It appears in the case of the rotorcraft that the non-linear inversion can be achieved more easily when the control structure is reduced to two layers. This is also the opportunity to extend this control law design technique to the case of non-affine systems.

Then the backstepping control approach, which has also been applied to airships[7] and aircraft [8,9] flight control law design, is introduced Two different design techniques are developed following the main guidelines of this approach. These two implementations appear of direct interest for the design of a new two-layer control structure based on backstepping control laws.

The third approach, differential flat control, has been already used to design generic guidance systems for aircraft $[10,11]$. After introducing some of the main relevant concepts of this already vast field, the partial differential flatness property of the fast rotorcraft dynamics is displayed. Since the slower dynamics present an affine structure, this induce to design first a differential flat control law for attitude, heading and altitude control and then, based on non-linear inversion, a control law for the horizontal track of the rotorcraft. The resulting control structure is quite similar to the one resulting from the backstepping one.

The three different control approaches are assessed and compared through a numerical simulation study while a quasi-linear proportional derivative multi-loop controller, tuned empirically, is also generated for reference purpose.

\section{Rotorcraft Flight Dynamics}

The considered system is shown in figure 1 where rotors one and three are clockwise while rotors two and four are counter clockwise. Annex 1 describes the rotor dynamics. The main simplifying assumptions adopted with respect to flight dynamics in this study are a rigid cross structure, constant wind, negligible aerodynamic contributions resulting from translational speed, no ground effect as well as small air density effects and negligible response times for the rotors. It is then possible to write the rotorcraft flight equations as follows [1].

The rotor forces and moments are given by:

$$
\begin{array}{cc}
F_{i}=f \omega_{i}{ }^{2} & i \in\{1,2,3,4\} \\
M_{i}=k F_{i}=k f \omega_{i}{ }^{2} & i \in\{1,2,3,4\}
\end{array}
$$

Where $f$ and $k$ are positive constants and $\omega_{i}$ is the rotational speed of rotor $i$. These speeds and forces satisfy the constraints:

$$
\begin{array}{ccrl}
0 & \leq \omega_{i_{i}} \leq \omega_{\max } & i \in\{1,2,3,4\} \\
0 \leq F_{i_{i}} \leq F_{i \max }=f \omega_{\max } & i \in\{1,2,3,4\}
\end{array}
$$

In Annex 1,the rotor dynamics are detailed. Since the inertia matrix of the rotorcraft can be considered diagonal with $I_{x x}=I_{y y}$, the roll, pitch and yaw moment equations may be written as:

$$
\dot{p}=\left(l\left(F_{4}-F_{2}\right)+k_{2} q r\right) / I_{x x}
$$




$$
\begin{gathered}
\dot{q}=\left(l\left(F_{1}-F_{3}\right)+k_{4} p r\right) / I_{y y} \\
\dot{r}=\left(k\left(F_{2}-F_{1}+F_{4}-F_{3}\right)\right) / I_{z z}
\end{gathered}
$$

Where $p, q$ and $r$ are the roll, pitch and yaw body angular rates. Here $k_{2}=\left(I_{z z}-I_{y y}\right)$ and $k_{4}=\left(I_{x x}-I_{z z}\right)$, where $I_{x x}$, $I_{y y}$ and $I_{z z}$ are the inertia moments in body-axis, and $l$ is the length of the four arms of the rotorcraft.

Let $\phi, \theta$ and $\psi$ be respectively the bank, pitch and heading angles, then the Euler equations relating the derivatives of the attitude angles to the body angular rates, are given by:

$$
\begin{gathered}
\dot{\phi}=p+\operatorname{tg}(\theta)(\sin \phi q+\cos \phi r) \\
\dot{\theta}=\cos \phi q-\sin \phi r \\
\dot{\psi}=(\sin \phi q+\cos \phi r) / \cos \theta
\end{gathered}
$$

In this study the wind is given in the local Earth reference frame by $\underline{w}=\left(\begin{array}{lll}w_{x} & w_{y} & w_{z}\end{array}\right)$. The wind is supposed constant while the ground effect is neglected. The acceleration $\underline{a}=\left(\begin{array}{lll}a_{x} & a_{y} & a_{z}\end{array}\right)^{\prime}$ of the centre of gravity, taken directly in the local Earth reference frame, is such as:

$$
\begin{gathered}
a_{x}=(1 / m)\left((\cos (\psi) \sin (\theta) \cos (\phi)+\sin (\psi) \sin (\phi)) F-d_{x}\right) \\
a_{y}=(1 / m)\left((\sin (\psi) \sin (\theta) \cos (\phi)-\cos (\psi) \sin (\phi)) F-d_{y}\right) \\
a_{z}=g-(1 / m)\left(\cos (\theta) \cos (\phi) F+d_{z}\right)
\end{gathered}
$$

where $x, y$ and $\mathrm{z}$ are the centre of gravity coordinates, $m$ is the total mass of the rotorcraft and:

$$
F=F_{1}+F_{2}+F_{3}+F_{4}
$$

Here the drag force $\underline{d}=\left(\begin{array}{lll}d_{x} & d_{y} & d_{z}\end{array}\right)^{\prime}$ is given by:

$$
\underline{d}=c \sqrt{\left(\dot{x}-w_{x}\right)^{2}+\left(\dot{y}-w_{y}\right)^{2}+\left(\dot{z}-w_{z}\right)^{2}}\left(\begin{array}{lll}
\dot{x}-w_{x} & \dot{y}-w_{y} & \dot{z}-w_{z}
\end{array}\right)^{\prime}
$$

with $c=1 / 2 \rho S C_{d}$ where $\rho$ is the volumetric mass of the air, $S$ is the reference surface for the aerodynamics of the rotorcraft and $C_{d}$ is its dimensionless drag factor. The components of the rotorcraft airspeed are:

$$
u_{a}=\dot{x}-w_{x}, \quad v_{a}=\dot{y}-w_{y} \quad \text { and } \quad w_{a}=\dot{z}-w_{z}
$$

The equilibrium conditions (hovering) in an horizontal wind $\left(w_{z}=0\right)$ with a given heading $\psi$ are such that:

$$
\begin{aligned}
& p=0, \quad q=0, \quad r=0, \quad \dot{x}=0, \quad \dot{y}=0, \quad \dot{z}=0 \\
& \theta_{e}=\operatorname{arctg}\left((c\|\underline{w}\| / m g)\left(\cos \psi \quad w_{x}+\sin \psi \quad w_{y}\right)\right) \\
& \phi_{e}=\operatorname{arctg}\left((c\|\underline{w}\| / m g)\left(\sin \psi w_{x}-\cos \psi w_{y}\right) \cos \theta_{e}\right) \\
& \text { with } \\
& F_{1}=F_{2}=F_{3}=F_{4}=m g /\left(4 \cos \theta_{e} \cos \phi_{e}\right)
\end{aligned}
$$

\section{A Reference Structure for Rotorcraft Flight Control}

Here we are interested in controlling the four-rotor aircraft so that its centre of gravity reaches and stays hovering at a predefined position while its heading acquires and maintains a given orientation. Many potential applications require this capability to be available in UAVs' while this problem can be also considered as a first step towards the design of more efficient trajectory tracking systems.

The manoeuvre under study is, when performed manually through direct radio control of the four engine thrusts (see picture 1), quite difficult to be achieved in one step. Experimentally it appears that no direct approach is feasible and that much depends on the rotorcraft attitude angles $\phi$ and $\theta$ specially when considering the control of its horizontal position error $\left(x-x_{c}, y-y_{c}\right)$.

Equations (5-1) and (5-2) show that to get any horizontal acceleration, it is necessary to have a non zero attitude ( $\phi \neq 0$ or $\theta \neq 0$ ), they show also that the orientation of the acceleration is dependent of the heading angle $\psi$. Equations (3-3) with (4-3) and (5-3) show that given the attitude angles $\phi$ and $\theta$, it is easy to master the heading 
angle error $\left(\psi-\psi_{c}\right)$ and the vertical position error $\left(z-z_{c}\right)$. From equations (3-1), (3-2) and (3-3), it appears that the effectiveness of the rotor actuators is much larger with respect to the roll and pitch axis than with respect to the yaw axis. Then we consider that attitude piloting is involved with controlling the angles $\theta$ and $\phi$. In equations (3-1) and (3-2), the effect of the rotor forces appears as differences so, we define new attitude inputs $u_{q}$ and $u_{p}$ as:

$$
u_{q}=F_{1}-F_{3} \quad u_{p}=F_{4}-F_{2}
$$

In the heading and position dynamics, the effects of rotor forces and moments appear as sums, so we define new guidance inputs $u_{\psi}$ and $u_{z}$ as:

$$
u_{\psi}=\left(F_{2}+F_{4}\right)-\left(F_{1}+F_{3}\right) \quad u_{z}=F=F_{1}+F_{2}+F_{3}+F_{4}
$$

Then

$$
\begin{aligned}
& \underline{F=T} \underline{\underline{u}} \\
& \text { with } T=\frac{1}{4}\left[\begin{array}{cccc}
0 & 2 & -1 & 1 \\
-2 & 0 & 1 & 1 \\
0 & -2 & -1 & 1 \\
2 & 0 & 1 & 1
\end{array}\right] \\
& \text { and } \quad \underline{F}=\left[\begin{array}{llll}
F_{1} & F_{2} & F_{3} & F_{4}
\end{array}\right]^{\prime} \quad \underline{u}=\left[\begin{array}{llll}
u_{p} & u_{q} & u_{\psi} & u_{z}
\end{array}\right]^{\prime}
\end{aligned}
$$

Equations (3-1), (3-2) and (3-3) are rewritten:

$$
\begin{gathered}
\dot{p}=\left(l u_{p}+k_{2} q r\right) / I_{x x} \\
\dot{q}=\left(l u_{q}+k_{4} p r\right) / I_{y y} \\
\dot{r}=k u_{\psi} / I_{z z}
\end{gathered}
$$

It appears that $u_{q}$ and $u_{r}$ can be made to vary significantly with $u_{\psi}$ and $u_{z}$ remaining constant. Attitude angles $\phi$ and $\theta$ can be seen as virtual controls for the horizontal position of the rotorcraft. Here the attitude dynamics are considered to be the fast dynamics, they are at the heart of the control system. The heading and height dynamics are intermediate while the dynamics of the horizontal position coordinates are the slower. This can lead to a three-level closed-loop control structure, as shown in figure 2, however the two inner or the two outer loops can be merged, if convenient, leading to different two-layer closed loop control structures, as will be seen in the next sections.

\section{Non-Linear Inverse Control of Rotorcraft Dynamics}

The basic idea of nonlinear dynamic inversion is to transform a non-linear dynamic system into an equivalent controllable linear system through a change of coordinates by compensating the non-linear parts of the system dynamics. This leads to adopt as control objective a stable linear dynamics for the output error. A non-linear state feedback control law can be derived when a non-singularity condition is satisfied

\section{A. Generalized Non-Linear Inverse Control}

Consider now a non-linear dynamic system given by:

$$
\begin{gathered}
\underline{\dot{X}}=g(\underline{X}, \underline{U}) \\
\underline{Y}=h(\underline{X})
\end{gathered}
$$

where $\underline{X} \in \mathrm{R}^{n}, \underline{U} \in \mathrm{R}^{m}, \underline{Y} \in \mathrm{R}^{m}, g$ is a smooth vector field of $\underline{X}$ and $\underline{U}$ and $h$ is a smooth vector field of $\underline{X}$. The system has, with respect to each independent output $Y_{i}$, a relative degree $r_{i}\left(\sum_{i=1}^{m}\left(r_{i}+1\right) \leq n, i=1, \ldots, m\right)$ around the state $\underline{X}_{0}$ if the output dynamics can be written as:

$$
\left(\begin{array}{c}
Y_{1}^{\left(r_{1}+1\right)} \\
\vdots \\
Y_{m}^{\left(r_{m}+1\right)}
\end{array}\right)=\underline{A}(\underline{X})+B(\underline{X}, \underline{U})
$$


If $B(\underline{X}, \underline{U})_{m \times m}$ is invertible with respect to $\underline{U}$, a feedback control law such as:

$$
\underline{U}=B^{-1}(\underline{X})(\underline{v}-\underline{A}(\underline{X}))
$$

can be obtained. Here the new control input $\underline{v}=\left[v_{1}, \ldots, v_{m}\right]$ is chosen such as:

$$
v_{i}=Y_{d i}^{\left(r_{i}\right)}-\sum_{k=0}^{r_{i}-1} c_{i k}\left(Y_{i}^{(k)}-Y_{d i}^{(k)}\right) \quad i=1 \text { to } m
$$

where $Y_{d i}$ is the reference control input for the output dynamics. Then the dynamics of the tracking error given by $e_{i}=Y_{i}-Y_{d i} i=1$ to $m$, are such as:

$$
e_{i}^{\left(r_{i}\right)}+c_{i r_{i}-1} e_{i}^{\left(r_{i}-1\right)}+\cdots+c_{i 1} e_{i}^{(1)}+c_{i 0} e_{i}=0
$$

where the coefficients $c_{i k}$ can be chosen to make the output dynamics asymptotically stable and ensure the tracking of output $y_{i}$ towards the reference output $y_{d i}$. However the derived feedback control law works only if either no internal dynamics $\left(\sum_{i=1}^{m}\left(r_{i}+1\right)=n\right)$ are present or if the internal dynamics $\left(\sum_{i=1}^{m}\left(r_{i}+1\right)<n\right)$ are stable. To cope with the saturation of the actuators, the choice of the coefficients $c_{i k}$ should be the result of a trade-off between the characteristics of the transient dynamics of the different outputs and the solicitations of the inputs.

\section{B. Rotorcraft Non-Linear Inverse Control Structure}

The flight dynamics of the rotorcraft, equations (3-i),(4-i) and (5-i) $i=1$ to 3 , cannot be modelled directly as an affine system such as (13-1) and (13-2), however the whole equations can be split in two sets:

- one relative to the roll and pitch dynamics (equations (3-1),(3-2), (4-1) and (4-2)),

- one relative to the position coordinates and the heading dynamics (equations (3-3), (4-3), (5-1), (5-2) and (5-3)).

The first set of equations is decoupled from the second one except for the yaw rate $r$, however since the yaw rate should remain small since heading dynamics are rather slow, this coupling can be neglected in a first step. The first set of equations provides $\phi$ and $\theta$ as virtual inputs to the second set of equations. Moreover, it appears that both set of equations satisfy the generalized invertibility condition. In the case of position coordinates and heading dynamics, since the real inputs $\left(u_{\psi}\right.$ and $\left.u_{z}\right)$ and the virtual inputs $(\phi$ and $\theta)$ are tightly mixed, the invertibility condition can be satisfied easily if the corresponding set of equations is taken as a whole. Then the non-linear inverse control approach is applied in two steps:

- One relative to the $\phi$ and $\theta$ attitude dynamics.

- One relative to the position coordinates and heading dynamics.

This leads to the control structure displayed in figure 3 where the two outer loops of the control structure presented in figure 2 have been merged. The first control layer is devoted to attitude control while the second one is devoted broadly to guidance control.

\section{Non-linear Inverse Attitude Control Layer}

The attitude dynamics can be rewritten under the form:

$$
\begin{gathered}
\underline{\dot{X}}=g\left(\underline{X}, \underline{U}, u_{z}\right) \\
\text { with here: } \quad \underline{X}=(p, \phi, q, \theta)^{\prime}, \underline{U}=\left(u_{p}, u_{q}\right)^{\prime} \text { and } \underline{Y}=(\theta, \phi)^{\prime}
\end{gathered}
$$

where $u_{\psi}$ is considered to be slowly varying with respect to the roll and pitch dynamics.

The attitude angles $\phi$ and $\theta$ have relative degrees equal to one and then there are no internal dynamics. The equations of the output dynamics can be rewritten as:

with

$$
\underline{Y}=M(\underline{Y}) \underline{U}+\underline{N}(\underline{Y}) u_{\psi}+\underline{P}(\underline{X}, r)
$$

$$
\begin{gathered}
M(\underline{Y})=\left[\begin{array}{cc}
l / I_{x x} & l \operatorname{tg} \theta \sin \theta / I_{y y} \\
0 & l \cos \phi / I_{y y}
\end{array}\right] \\
\underline{N}(\underline{Y})=\left[\begin{array}{c}
N_{p}(\underline{Y}) \\
N_{q}(\underline{Y})
\end{array}\right]=\left[\begin{array}{c}
k \operatorname{tg} \theta \cos \phi / I_{z z} \\
-k \sin \phi / I_{z z}
\end{array}\right] \\
\underline{P}(\underline{X}, r)=\left[P_{p}, P_{q}\right]^{\prime}
\end{gathered}
$$




$$
\begin{aligned}
& \text { where } P_{p}(\underline{X}, r)=k_{2} q r / I_{x x}+k_{4} \operatorname{tg} \theta \sin \phi p r / I_{y y}+r d(\operatorname{tg} \theta \cos \phi) / d t+q d(\operatorname{tg} \theta \sin \phi) \\
& \text { and } \\
& P_{q}(\underline{X}, r)=k_{4} \cos \phi p r / I_{y y}-q \sin \phi \dot{\phi}-r \cos \phi \dot{\phi}
\end{aligned}
$$

From (19-2), while $\phi \neq \pm \pi / 2$, the attitude dynamics given by (19-1) remain invertible. This leads to adopt as control objectives for the attitude angles $\phi$ and $\theta$, decoupled second order linear dynamics towards the current reference values $\phi_{c c}$ and $\theta_{c c}$ :

$$
\left[\begin{array}{l}
\ddot{\phi}_{d} \\
\ddot{\theta}_{d}
\end{array}\right]=\ddot{Y}_{d}=\left[\begin{array}{l}
-2 \zeta_{\phi} \omega_{\phi} \dot{\phi}-\omega_{\phi}^{2}\left(\phi-\phi_{c c}\right) \\
-2 \zeta_{\theta} \omega_{\theta} \dot{\theta}-\omega_{\theta}^{2}\left(\theta-\theta_{c c}\right)
\end{array}\right]
$$

where $\zeta_{\theta}, \zeta_{\phi}, \omega_{\theta}, \omega_{\phi}$ are respectively damping and natural frequency parameters while $\theta_{c c}$ and $\phi_{c c}$ are current reference values for the attitude angles, which are computed by the guidance controller.

Then the corresponding non-linear inverse attitude control law is given by:

$$
\begin{array}{cc} 
& {\left[\begin{array}{l}
u_{p} \\
u_{q}
\end{array}\right]=\underline{U}=-M(\underline{Y})^{-1}\left(\underline{N}(\underline{Y}) u_{\psi}+\underline{P}(\underline{X}, r)-\underline{Y}_{d}\right)} \\
\text { Then: } & u_{p}=\frac{I_{x x}}{l}\left(P_{p}-\ddot{\phi}_{d}+\frac{k \operatorname{tg} \theta}{I_{z z}}(\cos \phi+\operatorname{tg} \theta \sin \phi) u_{\psi}+\operatorname{tg}^{2} \theta\left(\ddot{\theta}_{d}-P_{q}\right)\right) \\
\text { and } & u_{q}=\frac{I_{y y}}{l \cos \phi}\left(2 \zeta_{\theta} \omega_{\theta} \dot{\theta}+\omega_{\theta}^{2}\left(\theta-\theta_{c}\right)+P_{q}-\frac{k \sin \phi}{I_{z z}} u_{\psi}\right)
\end{array}
$$

Then eliminating the second order terms, we get:

$$
\begin{aligned}
& u_{p}=\frac{I_{x x}}{l}\left(2 \varsigma_{\phi} \omega_{\phi} \dot{\phi}+\omega_{\phi}^{2}\left(\phi-\phi_{c}\right)+\frac{k \operatorname{tg} \theta}{I_{z z}} \cos \phi u_{\psi}\right) \\
& u_{q}=\frac{I_{y y}}{l \cos \phi}\left(2 \zeta_{\theta} \omega_{\theta} \dot{\theta}+\omega_{\theta}^{2}\left(\theta-\theta_{c}\right)-\frac{k \sin \phi}{I_{z z}} u_{\psi}\right)
\end{aligned}
$$

which are respectively linear and quasi-linear proportional derivative control laws. Observe that in (21-4) and (21-5) we have conserved the term with $u_{\psi}$ since it is, with respect to the attitude dynamics, a slow varying signal.

\section{Non-linear inverse guidance control Layer}

The outputs of the guidance dynamics are here $\psi, x, y$ and $z$. The output dynamics are then:

$$
\begin{aligned}
& \ddot{\psi}=k \cos \phi u_{\psi} /\left(\cos \theta I_{z z}\right)+l \sin \phi u_{p} /\left(\cos \theta I_{x x}\right)+g_{\psi} \\
& \ddot{x}=(1 / m)\left((\cos (\psi) \sin (\theta) \cos (\phi)+\sin (\psi) \sin (\phi)) u_{z}-d_{x}\right) \\
& \ddot{y}=(1 / m)\left((\sin (\psi) \sin (\theta) \cos (\phi)-\cos (\psi) \sin (\phi)) u_{z}-d_{y}\right) \\
& \ddot{z}=g-(1 / m)\left(\cos (\theta) \cos (\phi) u_{z}+d_{z}\right) \\
& \text { where } g_{\psi}=p d\left(\frac{\sin \phi}{\cos \theta}\right) / d t+r d\left(\frac{\cos \phi}{\cos \theta}\right) / d t+k_{2} \frac{q r \sin \phi}{\cos \theta I_{x x}}
\end{aligned}
$$

and $\underline{d}$ is given by (7).

The term $g_{\psi}$ is neglected since it remains very small during normal operations, moreover, its presence in equation (22-1) should turn difficult the computation of the current reference values for $\phi$ and $\theta$. The coupling term with the inner control loop, which remains small, is also removed from equation (22-1). Then considering the output equations (22-1), (22-2), (22-3) and (22-4), here also, the guidance dynamics presents, for each of its outputs, relative degrees equal to 1 while the internal dynamics, which are concerned with the attitude angles, are supposed already stabilized. Then, here also we are led to adopt, for $\psi, x, y$ and $z$, decoupled second order linear dynamics towards their target values $\psi_{c}, x_{c}, y_{c}$ and $z_{c}$ : 


$$
\left[\begin{array}{c}
\ddot{\psi}_{d} \\
\ddot{x}_{d} \\
\ddot{y}_{d} \\
\ddot{z}_{d}
\end{array}\right]=\left[\begin{array}{c}
-2 \zeta_{\psi} \omega_{\psi} \dot{\psi}-\omega_{\psi}{ }^{2}\left(\psi-\psi_{c}\right) \\
-2 \zeta_{x} \omega_{x} \dot{x}-\omega_{x}{ }^{2}\left(x-x_{c}\right) \\
-2 \zeta_{y} \omega_{y} \dot{y}-\omega_{y}{ }^{2}\left(y-y_{c}\right) \\
-2 \zeta_{z} \omega_{z} \dot{z}-\omega_{z}{ }^{2}\left(z-z_{c}\right)
\end{array}\right]
$$

where $\zeta_{\psi}, \zeta_{x}, \zeta_{y}, \zeta_{z}, \omega_{\psi}, \omega_{x}, \omega_{y}, \omega_{z}$ are respectively damping and natural frequency parameters, $\psi, x, y$ and $z$ and are the current values of the heading and the coordinates of the centre of gravity of the rotorcraft while $\psi_{c}, x_{c}, y_{c}$ and $z_{c}$ are the final reference values for the heading angle and the position coordinates. Of course, many other schemes can be proposed to define desired accelerations at the guidance level.

Once the desired accelerations are made available (relations (24-1), (24-2), (24-3) and (24-4)), the set of equations (22-1), (22-2), (22-3) and (22-4) must be solved simultaneously with respect to $u_{\psi}, u_{z}, \phi_{c c}$ and $\theta_{c c}$. We get the solution of this set of equations in the following order:

We get first the current reference attitude angles by elimination:

$$
\begin{gathered}
\theta_{c c}=\operatorname{arctg}\left(\frac{\ddot{x}_{d}+\delta_{x}}{g-\ddot{z}_{d}-\delta_{z}} \cos \psi+\frac{\ddot{y}_{d}+\delta_{y}}{g-\ddot{z}_{d}-\delta_{z}} \sin \psi\right) \\
\phi_{c c}=\operatorname{arctg}\left(\cos \theta\left(\frac{\ddot{x}_{d}+\delta_{x}}{g-\ddot{z}_{d}-\delta_{z}} \sin \psi-\frac{\ddot{y}_{d}+\delta_{y}}{g-\ddot{z}_{d}-\delta_{z}} \cos \psi\right)\right)
\end{gathered}
$$

Here $\delta_{x}=d_{x} / m \quad \delta_{y}=d_{y} / m \quad \delta_{z}=d_{z} / m$. Then, the current values of controls values of the controls $u_{\psi}$ and $u_{z}$ can be computed:

$$
\begin{gathered}
u_{\psi}=\frac{I_{z z} \cos \theta}{k \cos \phi} \ddot{\psi}_{d} \\
u_{z}=\left(m\left(g-\ddot{z}_{d}\right)-d_{z}\right) /(\cos \phi \cos \theta)
\end{gathered}
$$

Here also we get quasi-linear control law of the proportional derivative class. The values of $\phi_{c c}$ and $\theta_{c c}$ must be returned to the attitude controller to compute $u_{p}$ and $u_{q}$ using relations (21-2) and (21-3).

\section{Backstepping Control of Rotorcraft Dynamics}

The backstepping technique is a rather recent non-linear control technique, which applies to cascaded systems. The main idea is to use intermediate state variables as virtual inputs to take advantage of the causality relations displayed by the cascaded state representation. The convergence of the output variables towards their target values is obtained by the construction, step by step, of an auxiliary Lyapunov function. This general idea can be developed in different ways, as it will be shown in the next sub-section.

The main interest of the backstepping approach is that the stability of the controlled system as well as the convergence of the outputs towards their reference values can be guaranteed without inducing, like in the case of the non-linear control approach, the decoupling of the outputs dynamics. Indeed, it can be considered that the decoupling of the outputs dynamics demands an additional effort from the control channels with then a higher possibility of saturation for the actuators, either in position or speed, resulting in downgraded performances. Finally, another advantage of this approach is that several matrices of parameters are introduced while constructing the control law, providing a large variety of possibilities to shape conveniently the outputs dynamics as well as the control signals.

A. Two Examples of Implementation of the Backstepping Technique

Consider a cascaded system whose state representation is given by:

$$
\begin{gathered}
\underline{\dot{x}}_{1}=\underline{x}_{2} \\
\underline{\dot{x}}_{2}=g\left(\underline{x}_{2}, \underline{U}\right)
\end{gathered}
$$

where $\underline{x}_{1} \in R^{n}, \underline{x}_{2} \in R^{n}$ are state variables and $\underline{u} \in R^{n}$ is the control input and $g$ is a smooth diffeomorphism with respect to $\underline{u}$. The control objective here is to design a control law such that the state $\underline{x}_{1}$ can be stabilized at $\underline{x}_{1 c}$. 
Here also, $\underline{x}_{2}$ can be regarded as a virtual control input for the dynamics of $\underline{x}_{1}$ while the dynamics of $\underline{x}_{2}$ are controlled by the real control input $\underline{U}$. Now, suppose that there exists a control law $\underline{x}_{2}=G\left(\underline{x}_{1}, \underline{x}_{1 c}\right)$ such that the dynamics of $\underline{x}_{1}$ can be stabilized at $\underline{x}_{1 c}$ while we can find a Lyapunov function $V_{1}\left(\underline{x}_{1}-\underline{x}_{1 c}\right)$, which satisfies the condition:

$$
\dot{V}_{1}\left(\underline{x}_{1}-\underline{x}_{1 c}\right)=\left(\partial V_{1} / \partial \underline{x}_{1}\right)^{\prime} G\left(\underline{x}_{1}, \underline{x}_{1 c}\right) \leq-W\left(\underline{x}_{1}-\underline{x}_{1 c}\right)
$$

where $W\left(\underline{x}_{1}-x_{1 c}\right)$ is a positive definite function of $\underline{x}_{1}$. A possible choice is:

$$
G\left(\underline{x}_{1}, \underline{x}_{1 c}\right)=-\Lambda\left(\underline{x}_{1}-\underline{x}_{1 c}\right)
$$

where $\Lambda$ is a positive definite symmetric matrix. Then in this case:

$$
V_{1}\left(\underline{x}_{1}-\underline{x}_{1 c}\right)=W\left(\underline{x}_{1}, \underline{x}_{1 c}\right)=\frac{1}{2}\left(\underline{x}_{1}-\underline{x}_{1 c}\right)^{\prime}\left(\underline{x}_{1}-\underline{x}_{1 c}\right)
$$

The whole dynamics can be expressed as:

$$
\underline{\dot{x}}_{1}=G\left(\underline{x}_{1}, \underline{x}_{1 c}\right)+\underline{z} \quad \text { and } \quad \underline{\dot{z}}=\underline{w}
$$

where

$$
\underline{z}=\underline{x}_{2}-G\left(\underline{x}_{1}, \underline{x}_{1 c}\right)
$$

and

$$
\underline{w}=g\left(\underline{x}_{2}, \underline{u}\right)-\left(\partial G / \partial \underline{x}_{1}\right) \underline{x}_{2}
$$

Then a candidate Lyapunov function of the full system is given by:

$$
V\left(\underline{x}_{1}-\underline{x}_{1 c}, \underline{z}\right)=V_{1}\left(\underline{x}_{1}-\underline{x}_{1 c}\right)+1 / 2 \underline{z^{\prime}} \underline{z}
$$

The time derivative of $V\left(\underline{x}_{1}-\underline{x}_{1 c}, \underline{z}\right)$ is given by:

$$
\begin{aligned}
& \dot{V}\left(\underline{x}_{1}-\underline{x}_{1 c}, \underline{z}\right)=\left(\partial V_{1} / \partial \underline{x}_{1}\right)^{\prime}\left(G\left(\underline{x}_{1}, \underline{x}_{1 c}\right)+\underline{z}\right)+\underline{z}^{\prime} \underline{w} \\
\text { then: } \quad & \dot{V}\left(\underline{x}_{1}-\underline{x}_{1 c}, \underline{z}\right) \leq-W\left(\underline{x}_{1}-\underline{x}_{1 c}\right)+\left(\partial V_{1} / \partial \underline{x}_{1}\right)^{\prime} \underline{z}+\underline{z^{\prime}} \underline{w}
\end{aligned}
$$

and by an adequate choice of $\underline{w}$, such as:

$$
\underline{w}=-\left(\partial V_{1} / \partial \underline{x}_{1}\right)-\Omega \underline{z}
$$

where $\Omega$ is a symmetric positive definite matrix, the full system is globally asymptotically stable since it satisfies the following condition:

$$
\dot{V}\left(\underline{x}_{1}-\underline{x}_{1 c}, \underline{z}\right) \leq-W\left(\underline{x}_{1}-\underline{x}_{1 c}\right)-\underline{z}^{\prime} \Omega \underline{z}
$$

Finally, the effective control input is given by:

$$
\underline{U}=-g^{-1}\left(\underline{x}_{2}\right)\left(\left(\partial V_{1} / \partial \underline{x}_{1}\right)+\Omega\left(\underline{x}_{2}-G\left(\underline{x}_{1}, \underline{x}_{1 c}\right)\right)\right)
$$

Now we consider the case where the cascaded system cannot be written easily in the form (26-1) and (26-2), but its outputs obey to relations (13-2) and (14) with $r_{1}=r_{2}=\cdots=r_{m}=1$, where $B$ is invertible with respect to the control inputs. In that case, two auxiliary outputs can be defined:

$$
\underline{Z}_{1}=L\left(\underline{Y}-\underline{Y}_{c}\right)+\underline{\dot{Y}} \quad \text { and } \quad \underline{Z}_{2}=\underline{\dot{Y}}
$$

where $L$ is a positive definite symmetric matrix. A candidate Lyapunov function is then given by:

The time derivative of $V_{2}$ is such as:

$$
V_{2}=\frac{1}{2}\left(\underline{Z}_{1}^{\prime} \underline{Z}_{1}+\underline{Z}_{2}^{\prime} \underline{Z}_{2}\right)
$$

$$
\dot{V}_{2}=\underline{Z}_{1}^{\prime} \underline{\dot{Z}}_{1}+\underline{Z}_{2}^{\prime} \underline{\dot{Z}}_{2}
$$




$$
\dot{V}_{2}=\left(L\left(\underline{Y}-\underline{Y}_{c}\right)+2 \underline{\dot{Y}}\right)^{\prime}(L \underline{\dot{Y}}+\underline{Y})-L \underline{\dot{Y}}^{\prime} \underline{\dot{Y}}
$$

Choosing a control such as :

$$
L \underline{\dot{Y}}+\ddot{Y}=-\Lambda(L \underline{Y}+2 \underline{\dot{Y}})
$$

where $\Lambda$ is another symmetric positive definite matrix. We have:

$$
\dot{V}_{2}=-\left(L\left(\underline{Y}-\underline{Y}_{c}\right)+2 \underline{\dot{Y}}\right)^{\prime} \Lambda\left(L\left(\underline{Y}-\underline{Y}_{c}\right)+\underline{\dot{Y}}\right)-\underline{\dot{Y}}^{\prime} L \dot{Y}
$$

and it is straightforward to show that the system is globally asymptotically stable. The corresponding control law is given by (see (15)):

$$
\underline{U}=-B^{-1}(\underline{X})\left(\Lambda L\left(\underline{Y}-\underline{Y}_{c}\right)+(L+2 \Lambda) \underline{\dot{Y}}+\underline{A}(\underline{X})\right)
$$

Analyzing relations (3-i), (4-i) and (5-i), i =1 to 3, it appears that their equations can be separated into two sets: one relative to the slower dynamics, the horizontal dynamics, and corresponding to the first case considered in the previous section and one relative to other dynamics and corresponding to the second case. This leads to propose in figure 4 the following two layers control structure where, making reference to figure 2, the faster control loops have been merged.

\section{B. Attitude and Altitude Control by Backstepping}

The attitude and altitude dynamics are given by the state equations:

$$
\begin{gathered}
\dot{\phi}=p+\operatorname{tg}(\theta)(\sin \phi q+\cos \phi r) \\
\ddot{\phi}=\left(l u_{p}+l \operatorname{tg} \theta \sin \theta u_{q}+k \operatorname{tg} \theta \cos \phi u_{\psi}\right) / I_{x x}+P_{p}(p, q, r, \phi, \theta) \\
\dot{\theta}=\cos \phi q-\sin \phi r \\
\ddot{\theta}=\left(l \cos \phi u_{q}-k \sin \phi u_{\psi}\right) / I_{y y}+P_{q}(p, q, r, \phi, \theta) \\
\dot{\psi}=(\sin \phi q+\cos \phi r) / \cos \theta \\
\ddot{\psi}=k \cos \phi u_{\psi} /\left(\cos \theta I_{z z}\right)+l \sin \phi u_{p} /\left(\cos \theta I_{y y}\right)+g_{\psi}(p, q, r, \phi, \theta, \psi) \\
\dot{z}=v_{z} \\
\ddot{z}=g-(1 / m)\left(\cos (\theta) \cos (\phi) u_{z}+d_{z}\right)
\end{gathered}
$$

Here $P_{p}(p, q, r, \phi, \theta)$ and $P_{q}(p, q, r, \phi, \theta)$ are given respectively by expressions (19-5) and (19-6) while $g_{\psi}(p, q, r, \phi, \theta, \psi)$ is given by relation (23). The outputs dynamics (43-2), (43-4), (43-6) and (43-8) take the form (14) with $\underline{Y}=[\phi \theta \psi z]^{\prime}, \underline{U}=\underline{u}$ with $\underline{u}=\left(u_{p}, u_{q}, u_{\psi}, u_{z}\right)^{\prime}$. Here $\underline{X}=(p, q, r, \phi, \theta, \psi, \dot{z}, z)^{\prime}$ and $B(\underline{X}, \underline{u})$ is such as:

with

$$
B(\underline{X}, \underline{u})=J(\underline{Y}) \underline{u}
$$

$$
J(\underline{Y})=\left[\begin{array}{cccc}
\frac{l}{I_{x x}} & \frac{l \operatorname{tg} \theta \sin \theta}{I_{x x}} & \frac{k \operatorname{tg} \theta \cos \phi}{I_{x x}} & 0 \\
0 & \frac{l \cos \phi}{I_{y y}} & \frac{-k \sin \phi}{I_{y y}} & 0 \\
\frac{l \sin \phi}{\cos \theta I_{y y}} & 0 & \frac{k \cos \phi}{\cos \theta I_{z z}} & 0 \\
0 & 0 & 0 & \frac{-\cos \phi \cos \theta}{m}
\end{array}\right]
$$

and the above matrix is invertible if:

$$
\frac{\cos ^{2} \phi}{I_{z z}}-\frac{\operatorname{tg} \theta \sin \phi}{I_{y y}}\left(\sin \theta \sin \phi+\cos ^{2} \phi\right) \neq 0
$$

which is the case when $|\phi|$ and $|\theta|$ remain small with respect to $\pi / 2$.

We introduce now the two $R^{4 x 4}$ symmetric positive definite matrices $M$ and $\Lambda_{1}$ and adopt the control law (42),where:

$$
\underline{A}(\underline{X})=\left[\begin{array}{llll}
P_{p} & P_{q} & g_{\psi} & \left(g-d_{z} / m\right)
\end{array}\right]^{\prime}
$$


Since the actuator settings are determined by the inner control loop, let us have a look at the corresponding dynamics. The outputs $\phi, \theta, \psi$ and $z$ of the inner closed loop follows the dynamics given by:

$$
\left(\Lambda_{1} L\right)^{-1} \underline{Y}+\left(\Lambda_{1} L\right)^{-1}\left(L+2 \Lambda_{1}\right) \underline{\dot{Y}}+\left(\underline{Y}-\underline{Y}_{c}\right)=\underline{0}
$$

When matrices $\Lambda_{1}$ and $L$ are diagonal, these dynamics are decoupled and the poles of the decoupled dynamics are the roots of the $m$ different characteristic polynomials:

$$
\begin{gathered}
s^{2}+\left(\mu_{i}+2 \lambda_{1 i}\right) s+\lambda_{1 i} \mu_{i}=0 \quad i=1 \text { to } m \\
\text { where } \quad \Lambda_{1}=\operatorname{diag}\left(\lambda_{11}, \lambda_{12}, \cdots, \lambda_{1 m}\right) \text { and } L=\operatorname{diag}\left(\mu_{1}, \mu_{2}, \cdots, \mu_{m}\right)
\end{gathered}
$$

and where $s$ is the Laplace variable.

In this case, since the $\lambda_{l i}$ and the $\mu_{i}$ are positive real, we get always real negative roots. In the general case, the dynamics modes of the outputs will be characterized by the solutions of the global characteristic polynomial:

$$
\operatorname{det}\left(\left[\begin{array}{cc}
s I_{m} & -I_{m} \\
\Lambda_{1} L & s I_{m}+\left(L+2 \Lambda_{1}\right)
\end{array}\right]\right)=0
$$

Since this last relation is independent of the application it is possible to study once for all the reachable pole sets within the left half complex plane.

Remark: In the case of an horizontal wind, a necessary condition for final convergence and equilibrium, independent of the choice of matrices $\Lambda$ and $L$ or even of the control approach is given by:

$$
\begin{aligned}
& m g /\left(4 \cos \theta_{e} \cos \phi_{e}\right) \leq F_{i \max } \\
& \text { with } \quad \theta_{e}=\operatorname{arctg}\left((c\|\underline{w}\| / m g)\left(\cos \psi_{c} \quad w_{x}+\sin \psi_{c} \quad w_{y}\right)\right) \\
& \text { and } \quad \phi_{e}=\operatorname{arctg}\left((c\|\underline{w}\| / m g)\left(\sin \psi_{c} \quad w_{x}-\cos \psi_{c} \quad w_{y}\right) \cos \theta_{e}\right)
\end{aligned}
$$

\section{Horizontal Backstepping Control Layer}

The state representation of the horizontal dynamics is given by:

$$
\begin{gathered}
\dot{x}=v_{x} \\
\dot{y}=v_{y} \\
\dot{v}_{x}=(1 / m)\left((\cos (\psi) \sin (\theta) \cos (\phi)+\sin (\psi) \sin (\phi)) F-d_{x}\right) \\
\dot{v}_{y}=(1 / m)\left((\sin (\psi) \sin (\theta) \cos (\phi)-\cos (\psi) \sin (\phi)) F-d_{y}\right)
\end{gathered}
$$

where $\psi$ and $F$ are defined by the inner control loop.

This state representation corresponds to the one studied in the case of (26-1) and (26-2). Then following the corresponding backstepping approach, we get with $V_{l}$ chosen according to relation (29):

$$
\begin{gathered}
\phi_{c c}=\arcsin \left(\left(\sin \psi\left(m \varepsilon_{x}+d_{x}\right)-\cos \psi\left(m \varepsilon_{y}+d_{y}\right)\right) / u_{z}\right) \\
\theta_{c c}=\arcsin \left(\left(\cos \psi \frac{m \varepsilon_{x}+d_{x}}{u_{z}}+\sin \psi \frac{m \varepsilon_{y}+d_{y}}{u_{z}}\right) / \cos \phi_{c c}\right)
\end{gathered}
$$

where $\varepsilon_{x}$ and $\varepsilon_{y}$ are given by:

$$
\left[\begin{array}{c}
\varepsilon_{x} \\
\varepsilon_{y}
\end{array}\right]=-\left(I_{2}+\Omega \Lambda_{2}\right)\left[\begin{array}{l}
x-x_{c} \\
y-y_{c}
\end{array}\right]-\Omega\left[\begin{array}{c}
\dot{x} \\
\dot{y}
\end{array}\right]
$$

where $\Lambda_{2}$ and $\Omega$ are symmetric positive definite matrices. Then, the horizontal position of the rotorcraft follows the linear dynamics:

$$
\left[\begin{array}{c}
\ddot{x} \\
\ddot{y}
\end{array}\right]+\Omega\left[\begin{array}{c}
\dot{x} \\
\dot{y}
\end{array}\right]+\left(I_{2}+\Omega \Lambda_{2}\right)\left[\begin{array}{l}
x-x_{c} \\
y-y_{c}
\end{array}\right]=\underline{0}
$$

\section{Differential Flat Control for Rotorcraft Dynamics}

In the field of Robotics many control problems present strong non-linearities, which are not tractable through linearization. This has driven researchers to develop the notion of differential flatness for continuous non-linear 
dynamical systems. This notion, which has some similarity with both controllability and observability for linear systems, has open the door to a new approach for the design of non linear control laws. Many applications in the field of aerospace have already been performed with success. In this section, the differential flatness property of rotorcraft attitude and altitude dynamics taken as a whole, is displayed and a two layer control structure is proposed: one layer makes use of to differential flat control approach to provide a common control loop for attitude and altitude dynamics, and the other one provides, through non linear inversion, a control loop for the horizontal coordinates of the rotorcraft.

\section{A. Differential flatness of smooth systems}

A general nonlinear system given by:

$$
\underline{\dot{X}}=\varphi(\underline{X}, \underline{U}), \underline{X} \in R^{n}, \underline{U} \in R^{m}
$$

where $\varphi$ is a smooth mapping, is said explicitly flat with respect to the output vector $\underline{Z}$, if $\underline{Z}$ is an $m^{\text {th }}$ order vector which can be expressed analytically as a function of the current state, the current input and its derivatives, while the state and the input vectors can be expressed analytically as a function of $\underline{Z}$ and a finite number of its derivatives. Then there exists smooth mappings $G_{X}, G_{U}$, and $G_{Z}$ such as:

$$
\begin{gathered}
\underline{Z}=G_{Z}\left(\underline{X}, \underline{U}, \ldots, \underline{U}^{\left(n_{z}\right)}\right) \\
\underline{X}=G_{X}\left(\underline{Z}, \underline{\dot{Z}}, \ldots, \underline{Z}^{\left(n_{x}\right)}\right) \\
\underline{U}=G_{U}\left(\underline{Z}, \underline{Z}, \ldots, \underline{Z}^{\left(n_{x}+1\right)}\right)
\end{gathered}
$$

where $n_{z}$ and $n_{x}$ are integer numbers. Vector $\underline{Z}$ is called a flat output for the nonlinear system given by equation (54). Although until today there is no systematical way to determine flat outputs and eventually to prove its uniqueness, the flat outputs usually possess some physical meaning. The explicit flatness property is of particular interest for the solution of control problems when physically meaningful flat outputs can be related with their objectives. In many situations, the control problem can be formulated as a flat output trajectory following problem. In general, for these cases, the flat output of equation (55-1) can be reduced, through state transformation, to a function of a single argument, the new system state itself:

$$
\underline{Z}=G_{Z}(\underline{X})
$$

Then a possible control law providing to the flat outputs linear decoupled dynamics towards reference values is given by:

$$
\underline{U}=G_{U}\left(\underline{Z}, \underline{Z}, \ldots,-\sum_{i=1}^{n_{x}} A_{i} \underline{Z}^{(i)}-A_{0}\left(\underline{Z}-\underline{Z}_{c}\right)\right)
$$

where the $A_{i}$ matrices are diagonal matrices chosen such as the $m$ polynomials :

$$
s^{n_{x}+1}+\sum_{i=1}^{n_{x}} a_{i}(j, j) s^{i}+a_{0}(j, j) j=1, \ldots, m
$$

are characteristic polynomials corresponding to acceptable dynamics for the $m$ flat outputs.

\section{B. Differential flatness of rotorcraft dynamics}

The motion equations of the rotorcraft can be written in non-linear state form as: 


$$
\begin{array}{cc} 
& \dot{\dot{x}}=f(\underline{x}, \underline{u}) \\
\text { where } & \underline{x}=(p, q, r, \phi, \theta, \psi, \dot{x}, \dot{y}, \dot{z}, x, y, z)^{\prime} \\
\text { and } & \underline{u}=\left(u_{p}, u_{q}, u_{\psi}, u_{z}\right)^{\prime}
\end{array}
$$

Considering here the output vector $\underline{Z}=(\phi, \theta, \psi, z)^{\prime}$, the inversion of the Euler equations (4-1), (4-2) and (4-3) provides expressions such as:

or more specifically:

$$
\begin{aligned}
p & =p(\theta, \dot{\phi}, \dot{\psi}) \\
q & =q(\phi, \theta, \dot{\theta}, \dot{\psi}) \\
r & =r(\phi, \theta, \dot{\theta}, \dot{\psi})
\end{aligned}
$$

while $\underline{u}$ can be expressed as:

$$
\begin{gathered}
p=\dot{\phi}-\sin \theta \dot{\psi} \\
q=\cos \phi \dot{\theta}+\sin \phi \cos \theta \dot{\psi} \\
r=-\sin \phi \dot{\theta}+\cos \phi \cos \theta \dot{\psi}
\end{gathered}
$$

by inversion of the set of equations (3-1), (3-2), (3-3) and (5-3), or more specifically:

$$
\begin{gathered}
u_{p}=\left(-I_{x x} \dot{p}+k_{2} q r\right) / l \\
u_{q}=\left(I_{y y} \dot{q}-k_{4} p r\right) / l \\
u_{\psi}=-\left(I_{z z} \dot{r}\right) / k \\
u_{z}=-\left((\ddot{z}-g) m+d_{z}(\dot{x}, \dot{y}, \dot{z})\right) /(\cos \theta \cos \phi)
\end{gathered}
$$

Then, it can be concluded that the attitude and heading dynamics as well as the vertical dynamics of the rotorcraft are differentially flat when considering the input-output relation between $\underline{u}$ and $\underline{Z}$.

This leads to propose the control structure displayed in figure 5 which is close to the one proposed with the backstepping approach in figure 4 .

\section{Design of the differential flat control laws}

We adopt for the flat outputs second order dynamics and their second tie derivative should be such as:

$$
\begin{aligned}
& \ddot{\phi}_{d}=-2 \zeta_{\phi} \omega_{\phi} \dot{\phi}-\omega_{\phi}^{2}\left(\phi-\phi_{c c}\right) \\
& \ddot{\theta}_{d}=-2 \zeta_{\theta} \omega_{\theta} \dot{\theta}-\omega_{\theta}{ }^{2}\left(\theta-\theta_{c c}\right) \\
& \ddot{\psi}_{d}=-2 \zeta_{\psi} \omega_{\psi} \dot{\psi}-\omega_{\psi \psi}{ }^{2}\left(\psi-\psi_{c}\right) \\
& \ddot{z}_{d}=-2 \zeta_{z} \omega_{z} \dot{z}-\omega_{z}{ }^{2}\left(z-z_{c}\right)
\end{aligned}
$$

The expressions of the control inputs in relations (63-1), (63-2), (63-3) and (63-4) are fed by $p, q, r$ given by (61-1), (61-2) and (61-3) and by $\dot{p}, \dot{q}, \dot{r}$ given by:

$$
\begin{gathered}
\dot{p}=\ddot{\phi}_{d}-\cos \theta \dot{\theta} \dot{\psi}-\sin \theta \ddot{\psi}_{d} \\
\dot{q}=\cos \phi \ddot{\theta}_{d}+\sin \phi \cos \theta \ddot{\psi}_{d}-\sin \phi(1+\sin \theta) \dot{\theta} \dot{\psi}+\cos \phi \cos \theta \dot{\phi} \dot{\psi} \\
\dot{r}=-\sin \phi \ddot{\theta}_{d}+\cos \phi \cos \theta \ddot{\psi}_{d}-\cos \phi \dot{\phi} \dot{\theta}-\sin \phi \cos \theta \dot{\phi} \dot{\psi}-\cos \phi \sin \theta \dot{\theta} \dot{\psi}
\end{gathered}
$$

where $\ddot{\phi}_{d}, \ddot{\theta}_{d}, \ddot{\psi}_{d}$ and $\ddot{z}_{d}$ are given by (64-1), (64-2), (64-3) and (64-4) where appear the current target values for $\phi$ and $\theta, \phi_{c c}$ and $\theta_{c c}$, and the final target values of $\psi$ and $z, \psi_{c}$ and $z_{c}$. 


\section{Horizontal guidance by non-linear inverse control}

Now, considering equations (5-1) and (5-2), to insure that $x$ and $y$ adopt second order dynamics such as:

$$
\begin{aligned}
& \ddot{x}+2 \zeta_{x} \omega_{x} \dot{x}+\omega_{x}{ }^{2}\left(x-x_{c}\right)=0 \\
& \ddot{y}+2 \zeta_{y} \omega_{y} \dot{y}+\omega_{y}{ }^{2}\left(y-y_{c}\right)=0
\end{aligned}
$$

following the non linear inverse control approach, $\phi_{c c}$ and $\theta_{c c}$ must be chosen such as:

$$
\begin{aligned}
& (1 / m)\left(\left(\cos \psi \sin \theta_{c c} \cos \phi_{c c}+\sin \psi \sin \phi_{c c}\right) u_{z}-d_{x}\right)+2 \zeta_{x} \omega_{x} \dot{x}+\omega_{x}^{2}\left(x-x_{c}\right)=0 \\
& (1 / m)\left(\left(\sin \psi \sin \left(\theta_{c c}\right) \cos \phi_{c c}-\cos \psi \sin \phi_{c c}\right) u_{z}-d_{y}\right)+2 \zeta_{y} \omega_{y} \dot{y}+\omega_{y}^{2}\left(y-y_{c}\right)=0
\end{aligned}
$$

Then :

$$
\begin{aligned}
\phi_{c c} & =\arcsin \left(m\left(\sin \psi D_{x}-\cos \psi D_{y}\right) / u_{z}\right) \\
\theta_{c c} & =\arcsin \left(m\left(\cos \psi D_{x}+\sin \psi D_{y}\right) /\left(u_{z} \cos \phi_{c}\right)\right.
\end{aligned}
$$

where

$$
\begin{aligned}
& D_{x}=\delta_{x}-2 \zeta_{x} \omega_{x} \dot{x}-\omega_{x}{ }^{2}\left(x-x_{c}\right) \\
& D_{y}=\delta_{y}-2 \zeta_{y} \omega_{y} \dot{y}-\omega_{y}{ }^{2}\left(y-y_{c}\right)
\end{aligned}
$$

So it appears that differential flat control and non-linear inverse control can be easily integrated within a same multilayer control structure.

\section{Rotorcraft Quasi-Linear Control}

In this section the three non-linear control laws designed for the positioning of the rotorcraft are simplified to get quasi-linear control laws. Then a multi loop proportional derivative control law with a simple non-linear gain is introduced.

\section{A. Quasi linearization of the non-linear control laws}

In the case of the non-linear inverse control approach the obtained control laws are already quasi linear (see relations (21-4), (21-5), (25-3) and (25-4)). We can write:

$$
\begin{aligned}
& \tilde{u}_{p}=\frac{I_{x x}}{l}\left(2 \varsigma_{\phi} \omega_{\phi} \dot{\phi}+\omega_{\phi}^{2}\left(\phi-\phi_{c}\right)+\frac{k \operatorname{tg} \theta}{I_{z z}} \cos \phi u_{\psi}\right) \\
& \tilde{u}_{q}=\frac{I_{y y}}{l \cos \phi}\left(2 \zeta_{\theta} \omega_{\theta} \dot{\theta}+\omega_{\theta}{ }^{2}\left(\theta-\theta_{c}\right)-\frac{k \sin \phi}{I_{z z}} u_{\psi}\right) \\
& \tilde{u}_{\psi}=-\frac{I_{z z} \cos \theta}{k \cos \phi}\left(2 \varsigma_{\psi} \omega_{\psi} \dot{\psi}+\omega_{\psi}{ }^{2}\left(\psi-\psi_{c}\right)\right) \\
& \tilde{u}_{z}=\frac{m\left(g-\delta_{z}\right)}{\cos \phi \cos \theta}+\frac{m\left(2 \varsigma_{z} \omega_{z} \dot{z}+\omega_{z}{ }^{2}\left(z-z_{c}\right)\right)}{(\cos \phi \cos \theta)}
\end{aligned}
$$

The linearization of relations (25-1) and (25-2) can be realized in two steps:

First equalizing the sine with its angle:

$$
\begin{gathered}
\widetilde{\phi}_{c c}=\cos \theta\left(\frac{\ddot{x}+\delta_{x}}{g-\ddot{z}-\delta_{z}} \sin \psi-\frac{\ddot{y}+\delta_{y}}{g-\ddot{z}-\delta_{z}} \cos \psi\right) \\
\widetilde{\theta}_{c c}=\frac{\ddot{x}+\delta_{x}}{g-\ddot{z}-\delta_{z}} \cos \psi+\frac{\ddot{y}+\delta_{y}}{g-\ddot{z}-\delta_{z}} \sin \psi
\end{gathered}
$$

and then considering that $z$ is already close to its final value $z_{c}$, and if the wind is considered to have negligible effects, expressions (71-1) and (71-2) become:

$$
\begin{aligned}
& \tilde{\theta}_{c c}=-\frac{1}{g}\left(\cos \psi\left(2 \varsigma_{x} \omega_{x} \dot{x}+\omega_{x}{ }^{2}\left(x-x_{c}\right)\right)+\sin \psi\left(2 \varsigma_{y} \omega_{y} \dot{y}+\omega_{y}{ }^{2}\left(y-y_{c}\right)\right)\right. \\
& \widetilde{\phi}_{c c}=\frac{\cos \theta}{g}\left(\left(2 \varsigma_{x} \omega_{x} \dot{x}+\omega_{n}{ }^{2}\left(x-x_{c}\right)\right) \sin \psi-\left(2 \varsigma_{y} \omega_{y} \dot{y}+\omega_{y}{ }^{2}\left(y-y_{c}\right)\right) \cos \psi\right)
\end{aligned}
$$


The linearization of the backstepping control laws (relations (42), (48.1), (48.2) and (48-3)) gives:

$$
\left[\begin{array}{l}
\bar{u}_{p} \\
\bar{u}_{q} \\
\bar{u}_{\psi} \\
\bar{u}_{z}
\end{array}\right]=-J^{-1}\left((\Lambda L)\left[\begin{array}{c}
\phi-\phi_{c} \\
\theta-\theta_{c} \\
\psi-\psi_{c} \\
z-z_{c}
\end{array}\right]+(L+2 \Lambda)\left[\begin{array}{c}
\dot{\phi} \\
\dot{\theta} \\
\dot{\psi} \\
\dot{z}
\end{array}\right]+\left[\begin{array}{l}
0 \\
0 \\
0 \\
g
\end{array}\right]\right)
$$

with

$$
J^{-1}=\left[\begin{array}{cccc}
\frac{I_{x x}}{l} & 0 & \frac{-I_{x x} I_{z z}}{k I_{y y}} \operatorname{tg} \phi & 0 \\
0 & \frac{I_{y y}}{l \cos \phi} & \frac{-k \sin \phi}{I_{y y}} & 0 \\
-\operatorname{tg} \theta \cos \theta I_{z z} & 0 & \frac{\cos \theta I_{z z}}{k \cos \phi} & 0 \\
0 & 0 & 0 & \frac{-m}{\cos \phi \cos \theta}
\end{array}\right]
$$

So here again we get quasi-linear proportional derivative control laws. With respect to the computation of the current target values for the bank and pitch angle, we get in a first step:

$$
\begin{aligned}
& \bar{\phi}_{c c}=\left(\sin \psi\left(m \varepsilon_{x}+d_{x}\right)-\cos \psi\left(m \varepsilon_{y}+d_{y}\right)\right) / u_{z} \\
& \bar{\theta}_{c c}=\left(\cos \psi \frac{m \varepsilon_{x}+d_{x}}{\bar{u}_{z}}+\sin \psi \frac{m \varepsilon_{y}+d_{y}}{\bar{u}_{z}}\right) / \cos \bar{\phi}_{c c}
\end{aligned}
$$

where $\varepsilon_{x}$ and $\varepsilon_{y}$ are linear expressions given by:

$$
\left[\begin{array}{l}
\varepsilon_{x} \\
\varepsilon_{y}
\end{array}\right]=-\left(I_{2}+\Omega \Lambda_{2}\right)\left[\begin{array}{l}
x-x_{c} \\
y-y_{c}
\end{array}\right]-\Omega\left[\begin{array}{c}
\dot{x} \\
\dot{y}
\end{array}\right]
$$

Then $\bar{u}_{z}$ in (74-1) and (74-2) can be approximated roughly by:

$$
\bar{u}_{z}=m g /(\cos \theta \cos \phi)
$$

The linearization of the differential flat control law (relations (63-1) to (63-4), (64-1) to (64-4) and (65-1) to (65-3)) leads to:

$$
\begin{gathered}
\hat{u}_{p}=\left(I_{x x} / l\right)\left(2 \varsigma_{\phi} \omega_{\phi} \dot{\phi}+\omega_{\phi}{ }^{2}\left(\phi-\hat{\phi}_{c c}\right)+\sin \theta\left(2 \varsigma_{\psi} \omega_{\psi} \dot{\psi} \dot{p}+\omega_{\psi}{ }^{2}\left(\psi-\psi_{c}\right)\right)\right. \\
\hat{u}_{q}=-\left(I_{y y} / l\right)\left(\operatorname { c o s } \phi \left(2 \varsigma_{\theta} \omega_{\theta} \dot{\theta}+\omega_{\theta}{ }^{2}\left(\theta-\hat{\theta}_{c c}\right)+\sin \phi \cos \theta\left(2 \varsigma_{\psi} \omega_{\psi} \dot{\psi}+\omega_{\psi}{ }^{2}\left(\psi-\psi_{c}\right)\right)\right.\right. \\
\hat{u}_{\psi}=\left(I_{z z} / k\right)\left(\sin \phi\left(2 \varsigma_{\theta} \omega_{\theta} \dot{\theta}+\omega_{\theta}{ }^{2}\left(\theta-\hat{\theta}_{c c}\right)\right)-\cos \phi \cos \theta\left(2 \varsigma_{\psi} \omega_{\psi} \dot{\psi}+\omega_{\psi}{ }^{2}\left(\psi-\psi_{c}\right)\right)\right) \\
\hat{u}_{z}=\frac{m\left(g-\delta_{z}\right)}{\cos \theta \cos \phi}+\frac{m\left(2 \varsigma_{z} \omega_{z} \dot{z}+\omega_{z}{ }^{2}\left(z-z_{c}\right)\right)}{\cos \theta \cos \phi} \\
\left.\hat{\phi}_{c c}=\frac{m}{\hat{u}_{z}}\left(\sin \psi D_{x}-\cos \psi D_{y}\right)\right) \\
\hat{\theta}_{c c}=\frac{m}{\hat{u}_{z}}\left(\cos \psi D_{x}+\sin \psi D_{y}\right) / \cos \hat{\phi}_{c c} \\
D_{x}=\delta_{x}-2 \zeta_{x} \omega_{x} \dot{x}-\omega_{x}{ }^{2}\left(x-x_{c}\right) \\
D_{y}=\delta_{y}-2 \zeta_{y} \omega_{y} \dot{y}-\omega_{y}{ }^{2}\left(y-y_{c}\right)
\end{gathered}
$$

\section{B. Quasi -linear proportional derivative controller}

From the previous sub-section it appears that non-linear inverse control and differential flatness produce very close control laws while the backstepping approach seems able to provide, depending of the choice of matrices $L \Lambda_{1} \Omega$ and $\Lambda_{2}$, control laws quite different. Anyway, all the control laws developed here demonstrate to be non-linear 
extensions of proportional derivative control laws. The main difference between the different approaches is relative to the derived control structures.

For comparison purpose, a simplistic quasi-linear multi-loop control structure has been produced. Its control laws are completely decoupled and are given

- Attitude control laws:

$$
\begin{gathered}
\breve{u}_{p}=K_{\phi}\left(\breve{\phi}_{c c}-\phi\right)+K_{p} p \\
\breve{u}_{q}=K_{\theta}\left(\breve{\theta}_{c c}-\theta\right)+K_{q} q
\end{gathered}
$$

- Heading control law:

$$
\breve{u}_{\psi}=K_{\psi}\left(\psi_{c}-\psi\right)+K_{r} r
$$

- Horizontal control laws:

Let us define the auxiliary variables $\breve{u}_{x}$ and $\breve{u}_{y}$ by:

$$
\begin{aligned}
& \breve{u}_{x}=K_{x}\left(x_{c}-x\right)+K_{\dot{x}} \dot{x} \\
& \breve{u}_{y}=K_{y}\left(y_{c}-y\right)+K_{\dot{y}} \dot{y}
\end{aligned}
$$

then, where $\psi$ is the current heading of the rotorcraft:

- Vertical control law:

$$
\begin{aligned}
& \breve{\phi}_{c c}=-\left(\begin{array}{lll}
\sin \psi & \breve{u}_{x}-\cos \psi & \breve{u}_{y}
\end{array}\right) \\
& \breve{\theta}_{c c}=-\left(\begin{array}{lll}
\cos \psi & \breve{u}_{x}+c \sin \psi & \breve{u}_{y}
\end{array}\right)
\end{aligned}
$$

$$
\breve{u}_{z}=\frac{K_{z}\left(z_{c}-z\right)+K_{\dot{z}}}{\cos \phi \cos \theta}
$$

This last control law is non linear and numerical simulation tests has shown that the introduction of its non linear factor improves in a noticeable way the guidance performances of the rotorcraft under this modified control law.

\section{Simulation Study}

A view of two identical rotorcraft is shown in picture 1. Their physical parameters have been adopted for the numerical simulation. In Annex 1, the main physical characteristics of this class of rotorcraft are reported. The values of the gains of the backstepping control law have been chosen, in a first attempt, which has given rather good results, identical to those of the quasi-linear control law. These values have been obtained by tuning empirically the gains of the quasi-linear control law until an acceptable behaviour has been obtained. Since the backstepping control law, although non-linear, has the same structure than the quasi-linear control law, it needed the same gain structure and so the same gains values have been tested for it. The selected gains are displayed on Table 1.

The selected dynamics for the attitude, the altitude, the heading and the horizontal position are second order linear dynamics characterized by their respective damping coefficient and natural frequency. These values are reported on Table 2.

Although the non linear inverse control approach and the differential flat control approach are based on different control structures, since there are driven by common attitude and output dynamics (see table 2), they produce at the end control laws which are very similar and have basically the same performance. So the produced figures display different time responses of the rotorcraft under either the quasi-linear control law, the backstepping control law or the non-linear inverse control law.

The comparison is performed in two stages: first, the responses of the rotorcraft to a step in attitude (either $\phi$ or $\theta$ ) for each control law, are evaluated. Then, for each control law, the responses of the rotorcraft to a step in position ( $x, y$ or $z$ ) are evaluated and compared. The evaluation of the inner attitude control loop is important since the guidance capability of the rotorcraft, an under actuated device, is directly dependent of the controllability of its attitude angles.

The results (see figures 6 to 11) show that the three control laws, in both levels, present equivalent performances. However, while the non linear inverse control law produces second order linear dynamics for the attitude angles and the position and heading outputs, the two other control laws, even the quasi linear control law, produce clearly non 
linear behaviours for these variables. In particular (figure 6), since the final convergences of the quasi linear and the backstepping control laws are rather slow, the non linear inverse solution can produce, for a same response time, a less input demanding solution. However, as shown in the following figures, other parameters settings may lead to responses where the quasi linear and the backstepping approaches are slightly superior.

Other simulation studies should be performed in particular to show clearly the advantage of using advanced nonlinear control law instead of empirical-intuitive ones. Also, the realisation of simulation studies should be of interest to explore the impact of actuator saturations on the flight domain and feasible manoeuvres of the rotorcraft.

\section{Conclusion}

In this communication the applicability of three non-linear control approaches to the positioning and orientation of a rotorcraft has been treated. Since this system is highly nonlinear, naturally unstable and rather underactuated, the design of a unique control law to perform safely the whole manoeuvre is not straightforward and a multilevel control structure must be conceived. So along the paper, different control structures, associated to the different non-linear control approaches considered, are produced. Moreover, it appears that the direct application of these non-linear control approaches is not possible:

- In the case of the non-linear inverse control approach, it is necessary to extend its applicability to systems given by a non-affine state representation to bring a non-linear inverse control solution to the horizontal guidance sub problem.

- In the case of the backstepping control approach, it is necessary to realize two different implementations of its guidelines to insure the internal stability as well as the global stability of the controlled system.

- In the case of the differential flat control approach, first it has been necessary to identify the differential flatness property of a part of the rotorcraft flight dynamics, and then it has been necessary to combine this approach with the non-linear inverse control approach to treat the horizontal guidance sub problem.

The original paper considered also the important issue of the possible saturation of the actuators and proposed in each of the three considered approaches, the design of a supervision layer to avoid the occurrence or at least to limit the effects of actuator saturations. These lengthy developments have not been included in the present paper by lack of space.

Note also that the three developed non-linear control laws assume that, contrarily to the quasi-linear control law, an estimate of the wind speed is available. Should this estimate be computed on board or communicated from the ground? A partial solution to this problem could be provided by the possible robustness of these control laws with respect to external perturbations. The robustness with respect to other aspects such as parameters uncertainty is also an important issue since the payload of the rotorcraft can present important variations considering its reduced weight and its possible different missions. This question will be treated and it is to be expected that, whatever the adopted control solution, additional terms should be introduced. Of course, when considering the reference quasi-linear proportional derivative control law, the addition of an integrator term is expected.

The positioning and orientation problem, which has been treated here, can be seen as a first step towards the design of an efficient trajectory tracking system for the rotorcraft. A first move in this direction should be the evaluation of the proposed control schemes when the coordinates of the target point change slowly following a given 3D trajectory. Then a second move should be to integrate in the control structure predictive components so that the delays resulting from the inner control loops can be compensated. One of the main results of the present study is to provide insights into this question.

Two other interesting but very complex problems related with flight control of a rotorcraft are the generation of minimum time trajectories and the generation of minimum energy trajectories for the positioning and orientation problem. These two problems can be of interest depending of the application, their solutions provide the envelope of the whole set of feasible positioning and orientation manoeuvres. Another problem which can be of interest in this field is related with the safe landing ("soft crash") of a rotorcraft when an engine has failed. The solution of these three problems should use different control and decision techniques from those displayed in this paper.

Finally, the effective realisation of the control solutions depends of the availability of accurate measurement systems dedicated to the estimation of the angular rates $p, q$ and $r$, the attitude and heading angles, $\phi, \theta$ and $\psi$, as well as the 
inertial speed and position components, $\dot{x}, \dot{y}, \dot{z}$ and $x, y$, z. This accuracy could be obtained through some ad hoc hybridization schemes to overcome the specific difficulties associated with this light and small flying platform.

\section{References}

${ }^{1}$ G. Hoffmann, Rajnarayan, D.G., Waslander, S. L., Dostal, D., Jang, J.S. and Tomlin, C. J., "The Standford Tetsbed of Autonomous Rotorcraft for Multi-Agent Control", $23^{\text {rd }}$ Digital Avionics Systems Conference, Salt Lake City, Nov. 2004.

${ }^{2}$ H. K. Khalil, "Nonlinear Systems", Prentice Hall, 3rd Ed., 2002.

${ }^{3}$ R.S. Sutton and Barto, A. G., "Reinforcement Learning: an Introduction", MIT Press, Cambridge, MA, 1998.

${ }^{4}$ S.N. Singh and Schy, A. A., "Nonlinear Decoupled Control Synthesis for Maneuvring Aircraft", Proceedings of the 1978 IEEE conference on Decision and Control, Piscataway, NJ, 1978.

${ }^{5}$ R.Ghosh and Tomlin,C. J., "Nonlinear Inverse Dynamic Control for Model-based Flight”, Proceeding of AIAA, 2000.

${ }^{6}$ R. Asep, R., Shen, T.J., Achaíbou, K. and Mora-Camino, F., An Application of the Non-Linear Inverse Technique to Flight-path Supervision and Control, Proceedings of the 9th International Conference of Systems Engineering, Las Vegas,NV, 1993.

${ }^{7}$ A. Bento Moutinho, "Modeling and Non-Linear Control for Airship Autonomous Flight"PhD Thesis, Universidade Técnica de Lisboa, Instituto Superior Técnico, Lisbon, Dec. 2007.

${ }^{8}$ J. Farrell, Sharma M. and M. Polycarpou, "Backstepping-based Flight Control with Adaptive Function Approximation", Journal of Guidance, Control and Dynamics, 28(6),pp1089-1102, Nov.-Dec. 2005.

${ }^{9}$ T. Miquel, "Contribution à la synthèse de lois de guidage relatif, approche non linéaire ». PhD. Thesis, Université Paul Sabatier, Toulouse, 2004.

10 W.C.Lu, Mora-Camino, F. and Achaibou, K. , "Flight Mechanics and Differential Flatness". Dincon 04, Proceedings of Dynamics and Control Conference, Ilha Solteira, Brazil, pp. 830-839, 2004.

${ }^{11}$ L.Lavigne, F. Cazaurang F. and Bergeon B., "Modelling of Longitudinal Disturbed Aircraft Model by Flatness Approach", AIAA Guidance, Navigation, and Control Conference and Exhibit, Texas Austin, USA, 2003.

\begin{tabular}{|l|l|}
\hline $\mathrm{K}_{\theta}=-2$ & $\mathrm{~K}_{\mathrm{q}}=-0.23$ \\
\hline $\mathrm{K}_{\phi}=-2$ & $\mathrm{~K}_{\mathrm{p}}=-0.23$ \\
\hline $\mathrm{K}_{\psi}=-0.02$ & $\mathrm{~K}_{\mathrm{r}}=-0.025$ \\
\hline $\mathrm{K}_{\mathrm{z}}=0.12$ & $\mathrm{~K}_{\dot{z}}=0.15$ \\
\hline $\mathrm{K}_{\mathrm{x}}=0.137$ & $\mathrm{~K}_{\dot{x}}=0.183$ \\
\hline $\mathrm{K}_{\mathrm{y}}=0.137$ & $\mathrm{~K}_{\dot{y}}=0.183$ \\
\hline
\end{tabular}

Table 1 Selected gains for control laws

\begin{tabular}{|l|l|}
\hline$\zeta_{\theta}=0.8$ & $\omega_{\theta}=10 \mathrm{rad} / \mathrm{s}$ \\
\hline$\zeta_{\phi}=0.8$ & $\omega_{\phi}=10 \mathrm{rad} / \mathrm{s}$ \\
\hline$\zeta_{\psi}=0.8$ & $\omega_{\psi}=2 \mathrm{rad} / \mathrm{s}$ \\
\hline$\zeta_{z}=0.8$ & $\omega_{z}=1.5 \mathrm{rad} / \mathrm{s}$ \\
\hline$\zeta_{x}=0.8$ & $\omega_{x}=1.5 \mathrm{rad} / \mathrm{s}$ \\
\hline$\zeta_{y}=0.8$ & $\omega_{y}=1.5 \mathrm{rad} / \mathrm{s}$ \\
\hline
\end{tabular}

Table 2 Selected dynamics parameters 


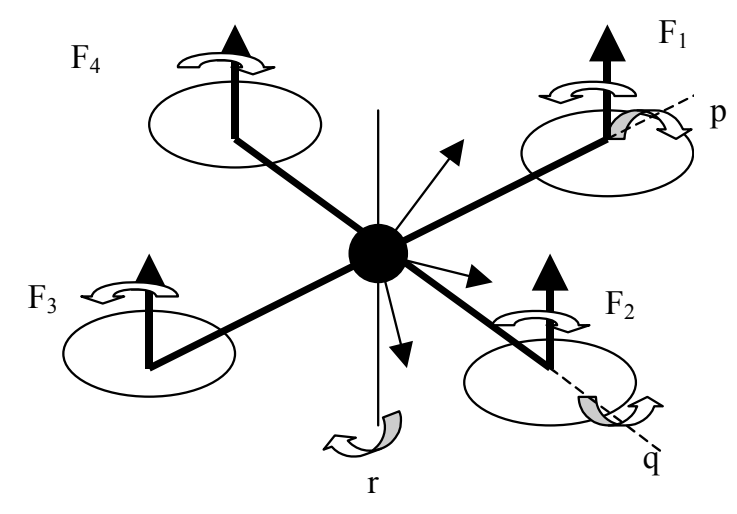

Figure 1 Rotorcraft Frame

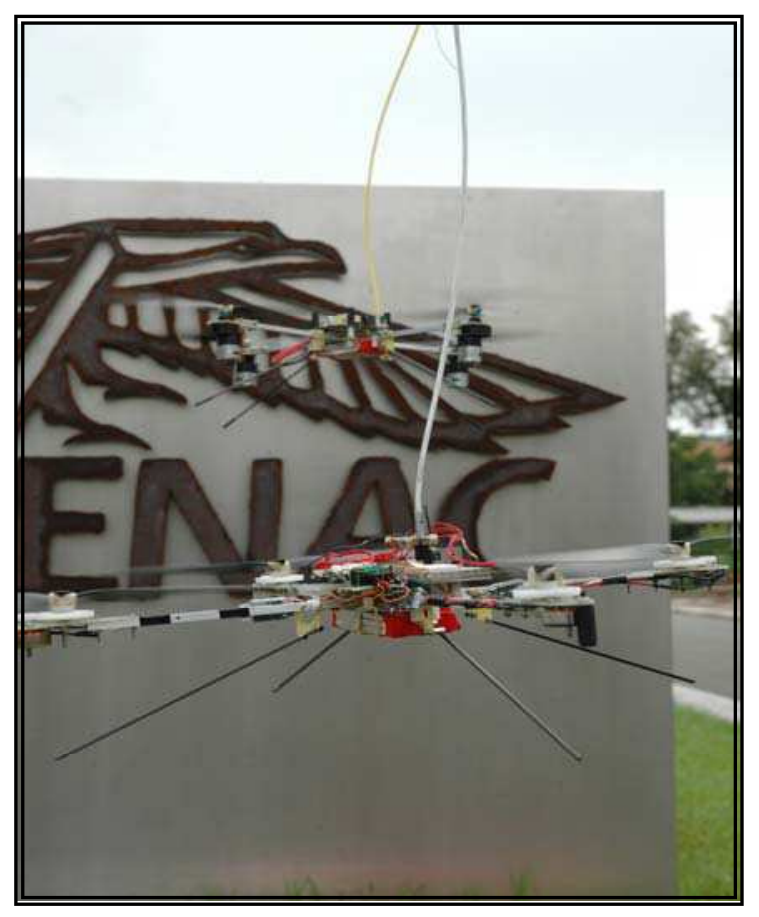

Picture 1 View of two hovering rotorcraft 


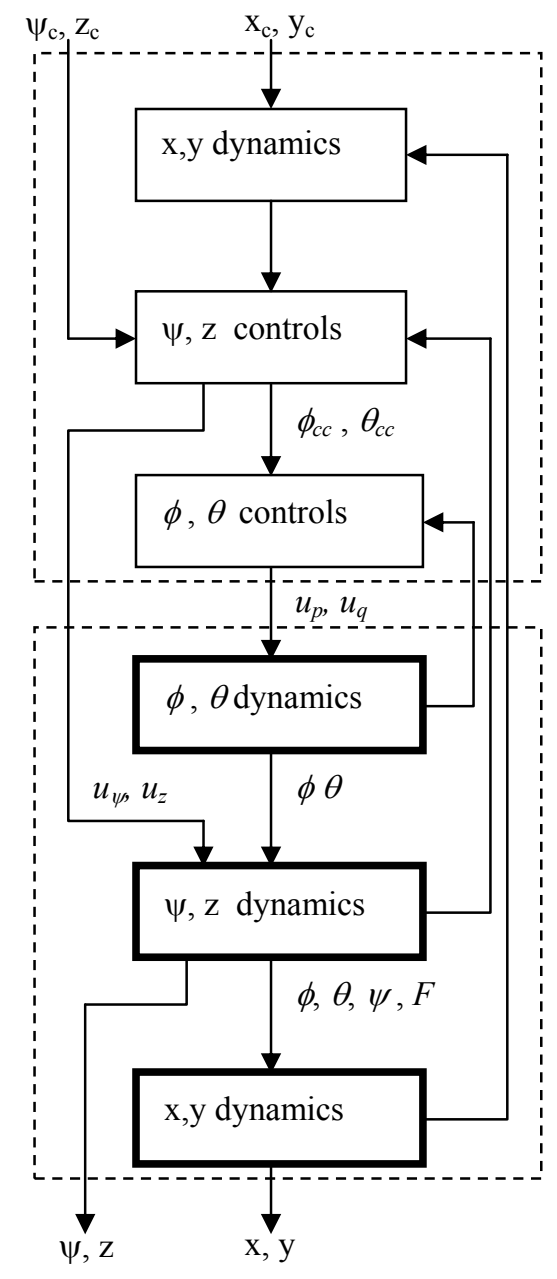

Figure 2. Three-layer rotorcraft dynamics control structure

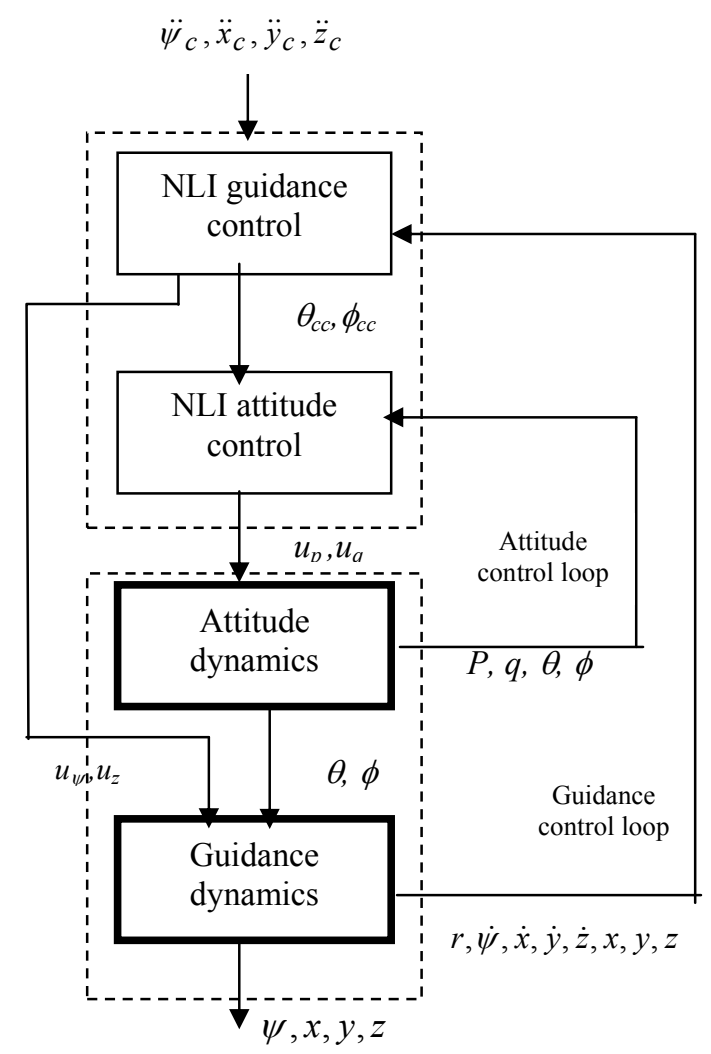

Figure 3 Two -layer non-linear inverse control structure 


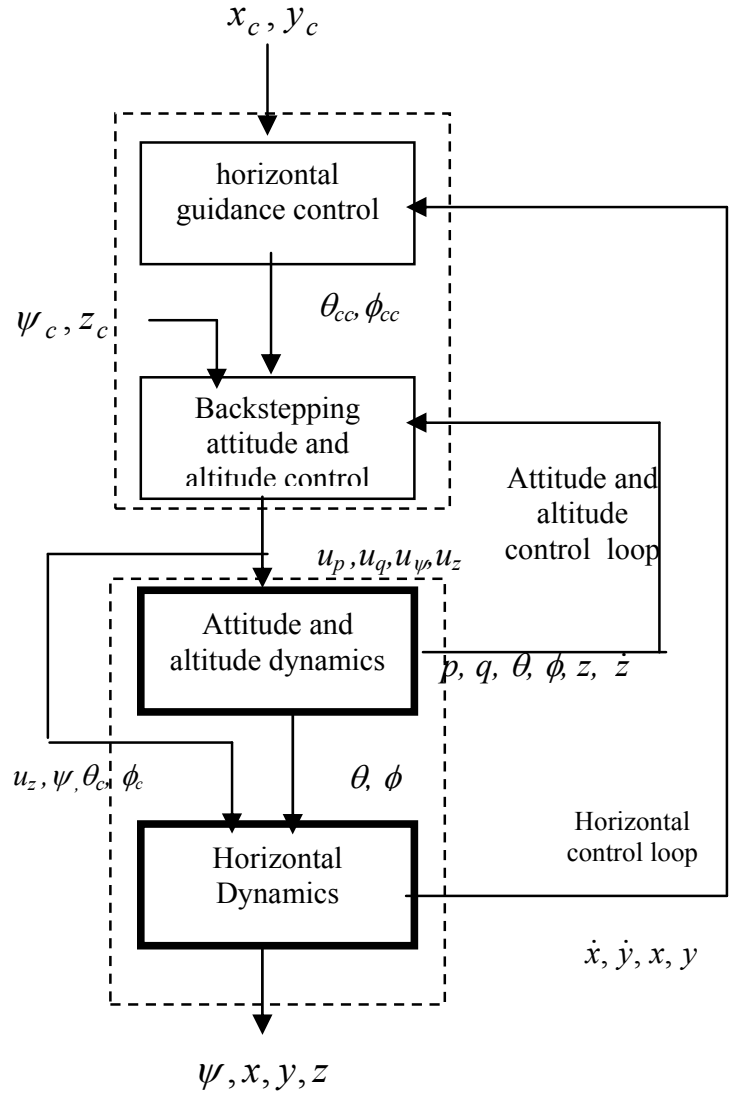

Figure 4 Two-layer backstepping control structure

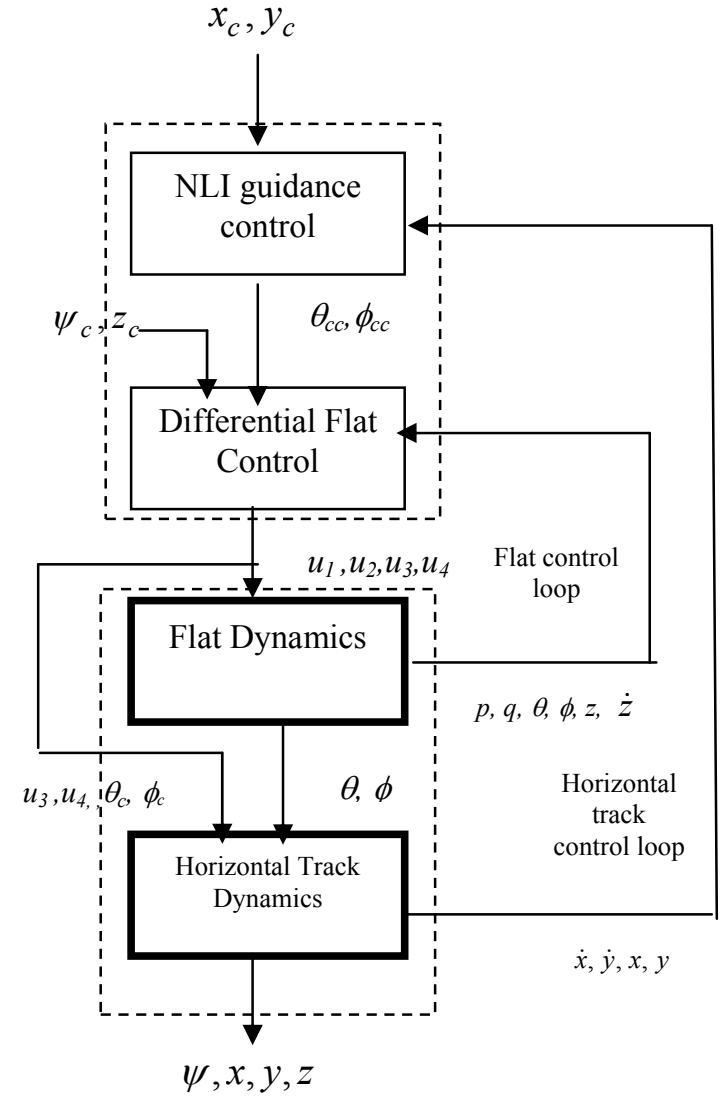

Figure 5 Differential flat-NLI control 

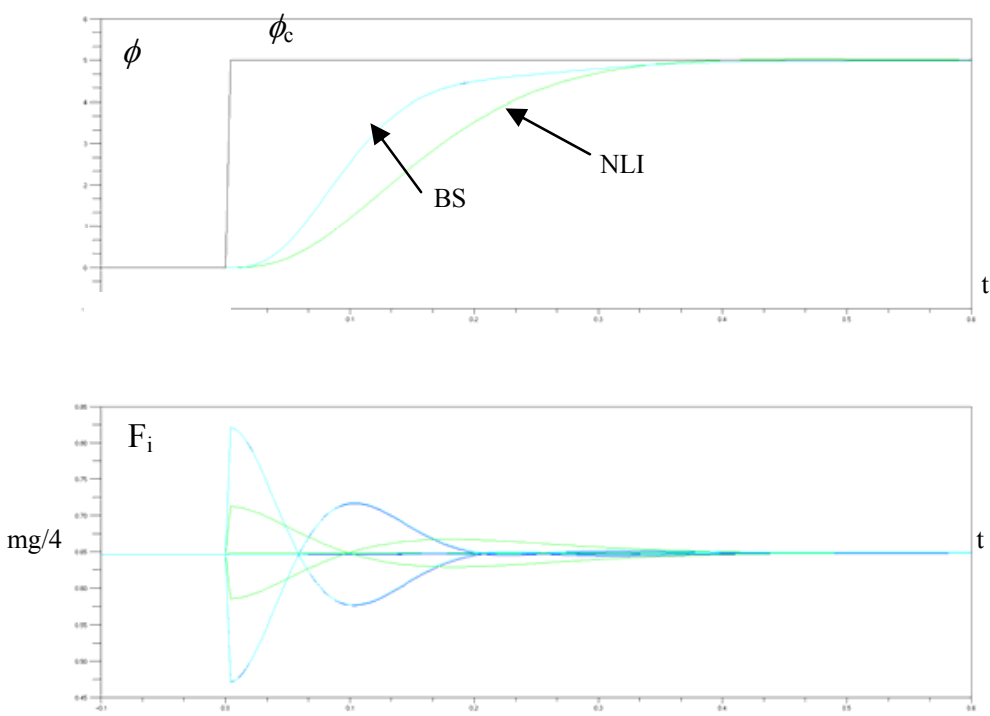

Figure 6 Step response of $\phi\left(\omega_{\phi}=10 \mathrm{rad} / \mathrm{s}\right)$

( NLI : non linear inverse, BS : backstepping )
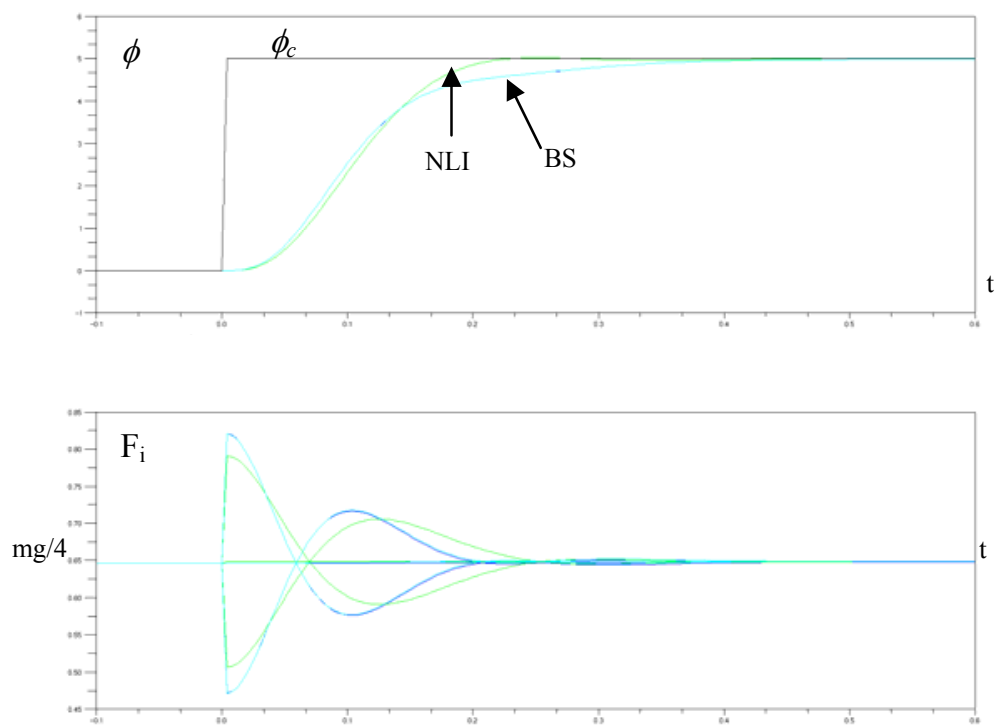

Figure 7 Step response of $\phi \quad\left(\omega_{\phi}=15 \mathrm{rad} / \mathrm{s}\right)$ 

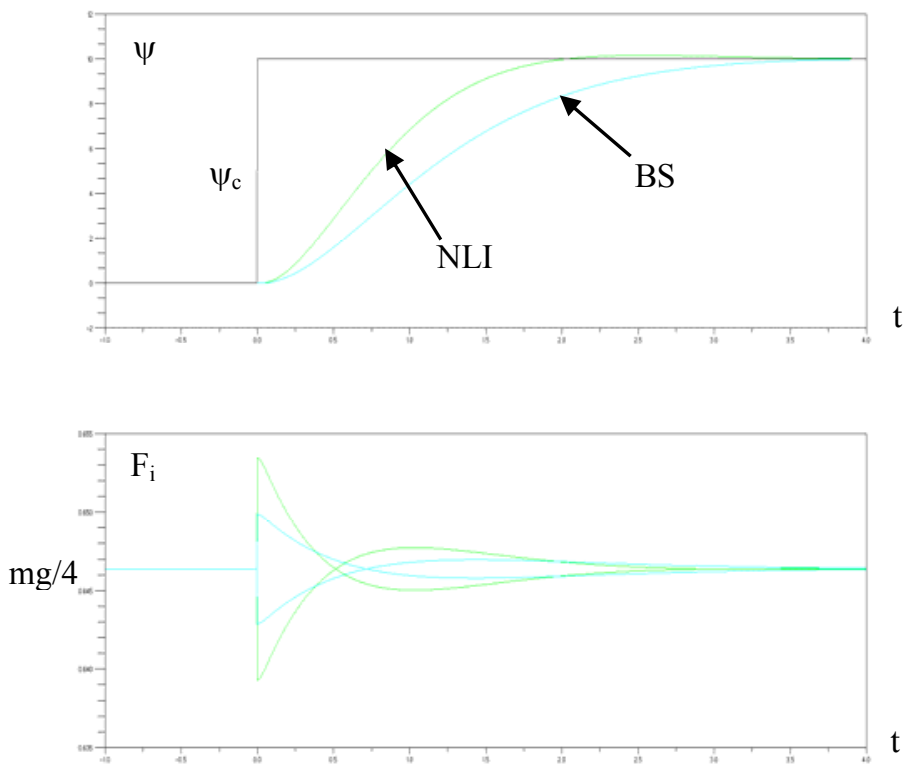

Figure 8 Step response of $\psi\left(\omega_{\psi}=5 \mathrm{rad} / \mathrm{s}\right)$
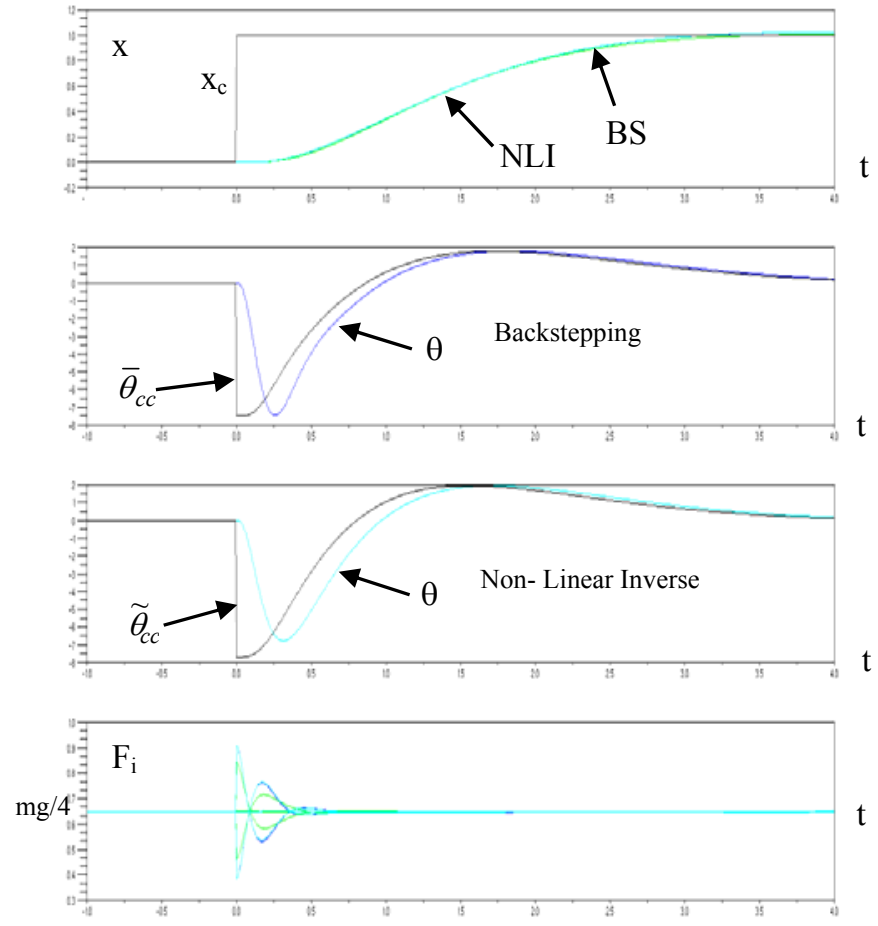

Figure 9 Step response of $\mathbf{x}$ with $\theta, \theta_{\mathbf{c c}} \quad\left(\omega_{\mathrm{x}}=1.5, \mathrm{rad} / \mathrm{s}, \omega_{\theta}=10 \mathrm{rad} / \mathrm{s}\right)$ 

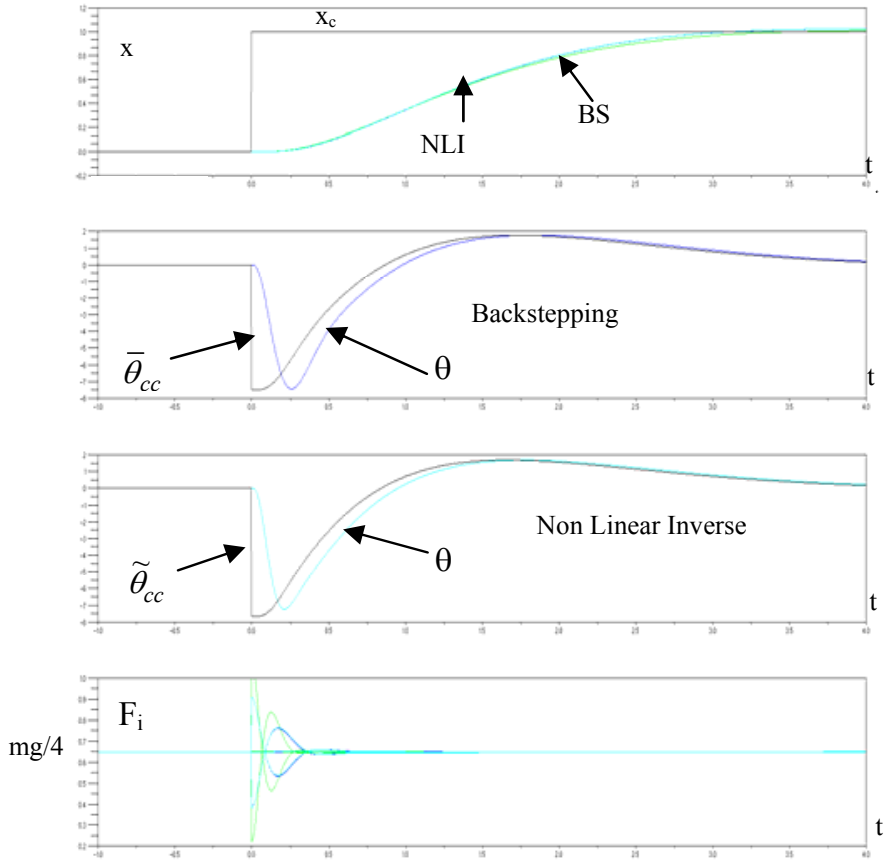

Figure 10. Step response of $\mathbf{x}$ (with $\theta, \theta_{\mathrm{c}}, \omega_{\mathrm{x}}=1.5, \mathrm{rad} / \mathrm{s}, \omega_{\theta}=15 \mathrm{rad} / \mathrm{s}$ )
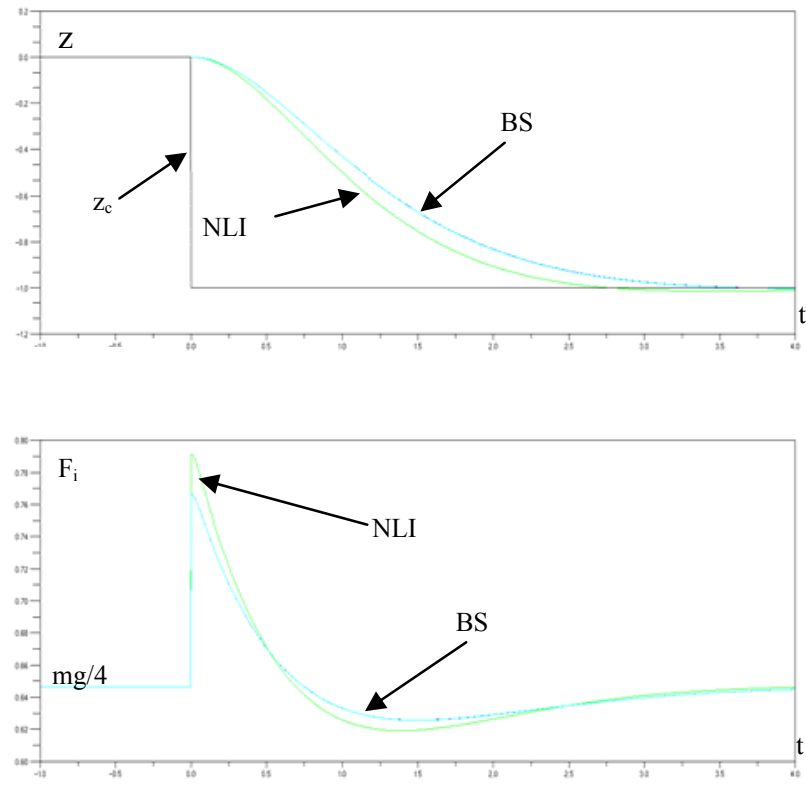

Figure 11 Step response of $z \quad\left(\omega_{z}=1.5 \mathrm{rad} / \mathrm{s}\right)$ 


\section{Appendix: Rotorcraft Physical Characteristics}

\section{Physical parameter values}

Mass: $m=500 \mathrm{~g}$, Body drag coefficient: $C_{d}=0.05$, Force coefficient: $f=0.5 \rho(0) S_{p} r_{p}{ }^{2} C_{t}$, Moment coefficient : $k=0.5 \rho(0) S_{p} r_{p}{ }^{2} C_{q}$, Volumetric mass of the air at see level : $\rho(0)=1.225 \mathrm{~kg} / \mathrm{m}^{3}$, Area of the propellers: $S_{p}=0.005 \mathrm{~m}^{2}$, Propeller radius: $r_{p=} 0.125 \mathrm{~m}$, Arms length: $l=0.25 \mathrm{~m}$, Propeller thrust aerodynamic coefficient: $C_{t}=0.297$, Propeller moment aerodynamic coefficient: $C_{q}=0.0276$, Inertia body axis coefficients: $I_{x x}=0.007 \mathrm{~kg} . \mathrm{m} 2$, $I_{y y}=0.0137 \mathrm{~kg} . \mathrm{m} 2, I_{z z}=0.0073 \mathrm{~kg} . \mathrm{m}^{2}$.

\section{Rotor engine dynamics}

The rotor engine dynamics are characterized by the relation between the input voltage $V_{a}$ and the angular rate $\omega$. A realistic model of rotor dynamics is given by:

$$
\dot{\omega}(t)=-\frac{1}{\tau} \omega(t)-K_{Q} \omega(t)^{2}+\left(K_{V_{a}} / \tau\right) V_{a}(t)
$$

where $\tau, K_{Q}$ and $K_{V a}$ are given positive parameters and where the voltage input is such as:

$$
0 \leq V_{a} \leq V_{\max }
$$

Numerical values for the rotors parameters: $\tau=10, K_{Q}=0.0079, K_{V a}=1000, V_{\max }=11 \mathrm{~V}$.

\section{Step response}

The step response ( $V_{a}=$ constant) of the rotor is solution of the scalar Riccati equation:

$$
\dot{\omega}(t)=-\frac{1}{\tau} \omega(t)-K_{Q} \omega(t)^{2}+\left(K_{V_{a}} / \tau\right) V_{a}
$$

with $\omega(0)=\omega_{0}$ A. particular solution $\omega_{1}$ of the associated differential equation is such as:

$$
\omega_{1}=\frac{1}{2 \tau K_{Q}}\left(\sqrt{1+4 K_{V_{a}} K_{Q} \tau V_{a}}-1\right)
$$

A particular solution $\omega_{1}$ of the associated differential equation is such as:

$$
\omega_{1}=\frac{1}{2 \tau K_{Q}}\left(\sqrt{1+4 K_{V_{a}} K_{Q} \tau V_{a}}-1\right)
$$

In the general case, the solution of (A.3) can be written as:

$$
\omega(t)=\omega_{1}+\frac{1}{\frac{1}{\omega(0)-\omega_{1}}+K_{Q} \tau^{\prime}\left(1-e^{-t / \tau^{\prime}}\right)} e^{-t / \tau^{\prime}}
$$

with

$$
\tau^{\prime}=\tau / \sqrt{1+4 K_{V} K_{Q} \tau V_{a}}
$$

and

$$
\lim _{t \rightarrow+\infty} \omega(t)=\omega_{1}
$$

It appears (see figure 12) that the dynamics of the rotor may be close to those of a first order linear system with time constant $\tau^{\prime}$, but as can be seen in (A.6), this value is a function of $V_{a}$. If the desired dynamics for the output are such as:

$$
\dot{\omega}=-(1 / T)\left(\omega-\omega_{c}\right)
$$

where $T$ is a very small time constant $V_{a}$ can be chosen such as: 


$$
V_{a}(t)=\frac{1}{K_{V_{a}}}\left(\left(1-\frac{\tau}{T}\right) \omega(t)+\frac{\tau}{T} \omega_{c}+\tau K_{Q} \omega(t)^{2}\right)
$$

Omega $(\mathrm{rad} / \mathrm{s}) \mathrm{Va}=2$
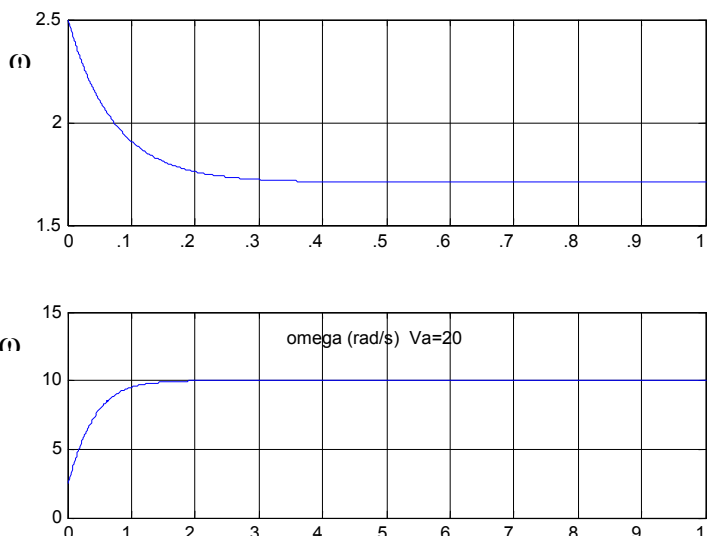

Figure 12 Two examples of rotor step response 